\title{
IL NUOVO ALLESTIMENTO DEL MUSEO DEL VICINO ORIENTE, EGITTO E MEDITERRANEO DELLA SAPIENZA
}

\author{
Lorenzo Nigro - Sapienza Università di Roma
}

\begin{abstract}
On March $19^{\text {th }} 2015$ the Museum of Near East, Egypt and Mediterranean was officially opened by our Magnificus Rector Prof. Eugenio Gaudio in a completely renewed exhibit in the magnificent seat of the Crescent Hall of Marcello Piacentini within the Palace of the Rectorate of Sapienza University. Works of art and archaeological items collected by expeditions working all over the Near East and the Mediterranean were displayed illustrating interconnections and differences among several cultures enlightening the long and rich history of these regions. The article explains the concept which inspired the renewed exhibit and illustrates the main highlights of the Near Eastern, Egyptian and Mediterranean collections.
\end{abstract}

Keywords: archaeology; cultural heritage; safeguard; management; restoration

\section{PRemessa: IL Museo del Vicino ORIENTE}

Il Museo del Vicino Oriente fu fondato da due autorevolissimi maestri della Sapienza, Sabatino Moscati e Sergio Donadoni, all'interno dell'Istituto del Vicino Oriente, la struttura che a partire dai primi anni Sessanta avrebbe raccolto un numero sempre maggiore di specialisti del Vicino Oriente antico e del Mediterraneo nello studio di discipline che spaziavano dalla Filologia semitica alle Lingue e alle Epigrafie semitiche, alla Storia del Vicino Oriente, l'Egittologia, l'Assiriologia, l'Ittitologia, l'Archeologia Orientale, Fenicia e Punica, conducendo ricerche innovative e per molti versi straordinarie in numerosi paesi del Vicino Oriente e del Mediterraneo e annoverando nei decenni successivi studiosi del calibro di Mario Liverani, Paolo Matthiae, Antonia Ciasca, Giovanni Garbini, Piero Bartoloni, Maria Giulia Amadasi ${ }^{1}$. L'Istituto del Vicino Oriente, sotto la fattiva direzione di Moscati fu, infatti, attivissimo nell'intraprendere missioni archeologiche in moltissimi paesi: Turchia, Siria, Libano, Israele, Cipro, Malta, Libia, Egitto, Sudan, Tunisia, Algeria, Marocco e - ovviamente - nelle due grandi isole italiane, la Sicilia e la Sardegna. Questa intensa attività sul campo, accompagnata da una altrettanto intensa attività di studio e pubblicazione nelle collane (Studi Semitici) e nelle riviste (Vicino Oriente) dell'Istituto, oltre che nelle serie fondate ad hoc per la pubblicazione dei risultati delle ricerche ad esempio a Malta, in Siria o in Sicilia, oltre a produrre straordinari risultati scientifici, condusse alla raccolta di una notevole quantità di reperti che, per diverse ragioni, furono portati a Roma.

Alla fine degli anni Cinquanta, le prime missioni condotte rispettivamente da Donadoni in Egitto (Tamit) e da Moscati in Israele (Ramat Rahel) avevano già raggruppato un numero considerevole di opere e reperti che formarono il primo nucleo significativo del Museo. Una svolta decisiva si ebbe, tuttavia, negli anni Sessanta, quando numerose nuove imprese si aggiunsero alle precedenti in Sicilia (Mozia, Pantelleria), Sardegna (Monte Sirai), Malta (Tas Silg̀), Siria (Ebla), Tunisia (Capo Bon, Ras ed-Drek), i primi divenendo cantieri di scavo stabili per i decenni successivi. La politica di queste missioni, anche per

1 Ciasca - Matthiae - Sist 1993. 
via dei loro giovani direttori, era fortemente innovativa e scevra da qualsiasi impronta coloniale. L'interesse eminentemente scientifico e le straordinarie relazioni diplomatiche intessute da Moscati e dai suoi allievi non prevedevano la collezione sistematica di reperti, tuttavia le eccellenti relazioni stabilite con i paesi nei quali venivano svolte le ricerche e circostanze derivanti dal forte impegno nel campo del restauro e della valorizzazione del patrimonio archeologico da parte delle missioni stesse portarono alla raccolta di materiali, concessi per studi archeometrici, per la realizzazione di repliche e, a volte, anche come segno della riconoscenza da parte degli stessi paesi ospiti. In alcuni casi, i reperti furono concessi liberalmente per ragioni di studio.

In parte diversa è la storia della formazione della Collezione Egizia. Essa fu costituita in massima parte da Donadoni durante gli scavi di salvataggio condotti a seguito della realizzazione della Diga di Assuan durante gli anni Sessanta. L'eccezionale recupero della Chiesa di Sonqi Tino, con le sue pitture parietali, gli scavi ad Antinoe, e, successivamente, i lavori di esplorazione e restauro nella Tomba di Sheshonq a Tebe portarono alla raccolta di un considerevole corpus di materiali, datanti dal periodo Protodinastico all'età islamica, che furono concessi dalla Repubblica Araba d'Egitto in virtù dello straordinario impegno profuso dalla Sapienza.

Ragioni simili, ossia derivanti dagli straordinari risultati conseguiti dalla Missione in Siria a Ebla e nei siti vicini di Tell Tuqan e Tell Afis, condotta da Paolo Matthiae, portarono alla realizzazione di una sala interamente dedicata a Ebla, con una cospicua collezione di frammenti ceramici, i modelli in scala del Palazzo degli Archivi e del Tempio di Ishtar sull'Acropoli, e, dopo la grande esposizione a Palazzo Venezia del 1995, del grande plastico della città paleosiriana della prima metà del II millennio a.C. Tra i pezzi più straordinari della collezione eblaita, sono le copie di diversi documenti cuneiformi (tra i quali il trattato tra Ebla e una non identificata città, primo esempio di trattato nella storia).

Così composto, nella sua seconda configurazione, il Museo del Vicino Oriente, magistralmente allestito dall'Arch. Carlo Cataldi Tassoni nella sede di Via Palestro, si articolava in due principali sezioni, quella siro-palestinese e quella egizia, cui si aggiungevano la sala di Ebla e una vetrina con i materiali punici di Mozia e di Monte Sirai.

\section{Il trasloco Del Museo, la sua Rifondazione nel Polo Museale E IL NuOvo ALLESTIMENTO DEL MVOEM}

Quando nel luglio del 2014 si è reso necessario lasciare la sede di Via Palestro, dove per trent'anni una parte considerevole del Dipartimento Scienze dell'Antichità (allora Dipartimento di Scienze Storiche, Archeologiche e Antropologiche dell'Antichità), inclusi il Museo del Vicino Oriente e diverse storiche missioni archeologiche, era stata allocata, il Magnifico Rettore Luigi Frati e il Consiglio di Amministrazione della Sapienza hanno identificato nel Palazzo del Rettorato una nuova sede per il Museo nella Sala a Crescente di Marcello Piacentini. Contestualmente, la creazione del Polo Museale Sapienza aveva fornito una rinnovata cornice istituzionale in cui inserire le attività del rinato museo ${ }^{2}$.

2 Al Magnifico Rettore Eugenio Gaudio, al Direttore Generale Carlo Musto D’Amore, al Pro-Rettore Gabriele Scarascia Mugnozza e al Direttore del Polo Museale Giorgio Manzi, nonché a tutti gli Uffici competenti di Sapienza, in particolare agli Architetti Claudio De Angelis e Giuseppe Luciani, vanno i più sentiti ringraziamenti per avere sostenuto i lavori del nuovo allestimento durante tutte le fasi di realizzazione. 
L'occasione del nuovo allestimento non solo ha consentito di riarticolare in parte le collezioni alla luce di nuove fondamentali acquisizioni (reperti dalla Sicilia, dalla Palestina, dalla Turchia, dalla Giordania e dall'Iraq), ma ha anche permesso di ripensare complessivamente il percorso espositivo, adattandolo alla nuova sede, ma anche conferendogli un taglio più al passo con i tempi, nel tentativo di realizzare una struttura in grado di rivolgersi sia al vasto pubblico, con una particolare attenzione rivolta ai bambini, sia agli studenti universitari.

Il trasloco è stato, inoltre, un'occasione unica per riesaminare dettagliatamente le collezioni, monitorare lo stato di conservazione delle opere, verificare la loro inventariazione (provenienza e status giuridico) ed annotarne le innumerevoli potenzialità scientifiche. Mentre le opere venivano imballate e nuovamente inventariate, un gruppo di giovani studiosi le ha potute riesaminare e fotografare. Sulla base di questo prezioso lavoro è stato costruito un nuovo percorso espositivo, suddiviso in trenta unità indipendenti, articolate in dodici temi maggiori, ma leggibili anche in un'unica sequenza che in modo coerente conduce dal Vicino Oriente all'Egitto, passando per l'intero Mediterraneo attraverso i millenni. Con l'eccellente collaborazione dell'ufficio tecnico di Sapienza e di un cospicuo numero di studenti e giovani studiosi è stato realizzato il nuovo allestimento.

La nuova concettualizzazione dell'esposizione, che intende valorizzare tutte le diversità di apporti culturali testimoniati nel Museo e le loro reciproche relazioni, ha portato con sé la nuova denominazione: Museo del Vicino Oriente, Egitto e Mediterraneo.

\subsection{Il concept del Museo VOEM: New light on the Near East, Egypt and the Mediterranean}

Dopo avere annunciato il nuovo nome del Museo e prima di descrivere più partitamente la nuova organizzazione delle Collezioni, mi sembra utile e necessario spendere due parole sulla nuova concezione dell'esposizione, che mira principalmente a ripartire dalle opere, dalla loro corretta contestualizzazione, lasciando al visitatore il piacere di percorrere gli innumerevoli itinerari culturali che lo straordinario spazio espositivo della Sala a Crescente di Marcello Piacentini, costellata di antichissime opere, suggerisce (fig. 1).

Il 2015 è stato un anno infausto per l'area geografica e culturale cui il Museo è dedicato e l'archeologia è stata vittima delle tragedie che hanno travolto e stanno travolgendo i popoli di tanti paesi del Vicino Oriente e del Mediterraneo e con loro anche deliberatamente e sciaguratamente - lo straordinario patrimonio archeologico che essi custodiscono. Aprire un nuovo Museo può, quindi, sembrare inutile, tanta è la sproporzione tra i passi in avanti nella ricerca e nella valorizzazione del patrimonio archeologico conseguiti dalle Missioni della Sapienza e le terribili distruzioni di cui siamo malauguratamente testimoni. Tuttavia, il Museo è per i giovani (bambini della Scuola Elementare e studenti dell'Università) e vuole essere una fiammella di speranza, quella piccola nuova luce che deve riaccendersi e vedere uniti tutte le donne e gli uomini del mondo nello scoprire, conservare, proteggere e valorizzare quel patrimonio di memoria condivisa di culture tra loro diverse e autonome.

Con l'aiuto di colleghi e studenti avanzati è stato dunque realizzato un unico progetto espositivo mirante all'illustrazione delle culture antiche del Mediterraneo osservate ciascuna nella propria autonomia e dignità, segnalandone allo stesso tempo le interconnessioni, le influenze reciproche, le straordinarie acquisizioni, ma anche i contrasti 
e i fallimenti, rappresentando quel mosaico di popoli e culture che hanno tutti spazio nella nostra eredità condivisa.

Un riferimento particolare, ovviamente, è andato alle spedizioni archeologiche che hanno riscoperto e studiato i centri dai quali i reperti provengono e che tanto hanno contribuito a tenere alto il nome della Sapienza e dell'Italia nell'archeologia mondiale.

\subsection{Articolazione delle collezioni e nuove acquisizioni}

Prima di progettare e realizzare il nuovo allestimento è stato necessario riorganizzare le collezioni, sia per valorizzare alcuni lotti di materiali non particolarmente considerati in passato, sia per conferire la giusta dignità alle nuove importanti acquisizioni. Sono state così costituite delle unità espositive ${ }^{3}$, conservando, per ragioni di chiarezza inventariale la bipartizione tra Collezione Vicino Orientale e Collezione Egizia.

Nella Collezione Vicino Orientale si annoverano i materiali dalla Turchia (Arslantepe); Palestina (Gerico) ${ }^{4}$, dalla Giordania (Khirbet al-Batrawy), dal Libano (Achziv), da Israele (Ramat Rahel), da Malta (Tas Silg; San Pawl Milqi, Ras al-Wardiyah), dalla Tunisia (Capo Bon, Areg el-Rhazouani, Kerkouane, Ras ed-Drek), da Pantelleria, dalla Sicilia (Mozia), dalla Sardegna (Monte Sirai). A questo corpus si aggiungono alcuni materiali da Cipro (Kition), dall’Algeria (Banasa) e dal Marocco (Mogador) ottenuti dal Prof. Moscati.

Nella Collezione Egizia, le opere provengono oltre che dagli scavi di Sonqi Tino, Arsinoe e Antinoe in Egitto; anche dalla missione al Jebel Barkal (Napata) e Mussawwarat es-Sufra in Sudan settentrionale, oltreché da numerose donazioni ottenute dal Prof. Donadoni.

Le novità ${ }^{5}$ sono costituite dal gruppo di reperti da Tell es-Sultan/antica Gerico in Palestina, con la riproduzione di due mattoni crudi (uno del tipo "loaf shaped" del Neolitico Preceramico A, l'altro “a sigaro” del Neolitico Preceramico B), una replica di uno dei teschi del Neolitico Pre-Ceramico B e un plastico in scala 1:20 della Torre Circolare del Neolitico Preceramico ${ }^{6}$; da un doppio set di repliche di importanti reperti rinvenuti dalla Missione archeologica in Turchia ad Arslantepe (uno composto da vasi e cretule stampigliate che sono tra le più perspicue testimonianze del funzionamento di un sistema amministrativo centrale in epoca protostorica nel Vicino Oriente; il secondo costituito da straordinarie copie del corredo di armi e ornamenti in argento, bronzo ed elettro, ritrovati nella tomba del principe della stessa città) ${ }^{7}$; dalle copie della coppa del re e delle asce di rame ritrovate dalla Missione archeologica in Palestina e Giordania nel Palazzo di Khirbet al-Batrawy ${ }^{8}$; da un’àncora litica ${ }^{9}$ e diversi altri reperti da Mozia; dalla copia assai rara della

3 Ciascuna unità è costituita da una o più vetrine, con i relativi cassetti che conservano i reperti pertinenti alla stessa unità/provenienza/area non esposti. Ciascuna vetrina infatti dispone da due a quattro cassetti posizionati sotto il piano espositivo.

4 La collezione include anche materiali da Tell el-'Areini, Tell el-Mafjar, Tell el-Matlab, Tell es-Samrat, Tell Sheikh Abu Zarad, Betlemme.

5 Per una descrizione più dettagliata e scientifica delle opere si rimanda alla prossima pubblicazione del Catalogo del Museo VOEM.

6 Rispettivamente, inv. nn. VO3000, VO3415.

7 Frangipane 1998.

8 Rispettivamente: inv. nn. VO3197, VO3207/I-IV.

9 Inv. n. M3410. 
statua non finita della regina Nefertiti ritrovata a el-Amarna ${ }^{10}$. Infine, in occasione dell'inaugurazione del Museo, il Regno Hashemita di Giordania ha donato al Museo una replica in scala ridotta del mosaico pavimentale del tepidarium del Hammam del Palazzo del Califfo Hisham (Khirbet el-Mafjar) a Gerico ${ }^{11}$, realizzata dalla Scuola di restauro del mosaico fondata dal compianto Padre Michele Piccirillo o.f.m. a Madaba.

Con l'occasione della nuova esposizione molti reperti sono stati restaurati grazie alla maestria e alla disponibilità di Salvatore Tricoli, a volte anche con interessanti esiti scientifici, come nel caso del rimontaggio della statua di personaggio divino itifallico (Baal Hammon) dal Tofet di Monte Sirai ${ }^{12}$.

\subsection{Il percorso espositivo e l'apparato illustrativo e didascalico}

Le due collezioni maggiori sono state disposte in sequenza ipotizzando un unico percorso di visita all'interno della sala, in senso orario. Tuttavia, la collezione egizia ha anch'essa un suo pannello introduttivo e può essere visitata separatamente. Sono state distinte quattro grandi aree tematiche attraverso una banda colorata che corre in alto sui pannelli illustrativi: verde = Vicino Oriente pre-classico; azzurro = Fenici e Mediterraneo; giallo ocra = Egitto faraonico; arancione $=$ Egitto copto e paleocristiano; violetto $=$ Egitto ellenistico e romano ${ }^{13}$.

L'esposizione è composta da unità espositive con i materiali disposti in trenta vetrine dotate di cassetti e cinque pedane aperte.

L'apparato didascalico illustrativo è composto dai seguenti elementi: a) dieci grandi pannelli tematici che introducono ciascuna sezione; b) trenta pannelli delle unità espositive collocali all'interno delle vetrine di due diverse dimensioni; c) didascalie dei singoli reperti (con luogo di rinvenimento, datazione e breve descrizione); d) sussidi informatici (attraverso il QR code che collega gli apparati con il sito web del Museo è possibile verificare la didascalia di ciascun reperto e leggere il testo dei pannelli); e) schermo touch screen per le attività didattiche con le scuole; f) roll up tematici disposti lungo il percorso.

Sono state individuate trenta unità espositive, due delle quali (nn. 15 e 30), una per ciascuna collezione maggiore, ha una concezione aperta e serve ad ospitare reperti messi in esposizione a rotazione.

\subsection{Le unità espositive}

Il percorso inizia con un piccolo pannello di credits, che illustra il concept del Museo e ricorda il fondamentale contributo offerto dal Ministero degli Affari Esteri e della Cooperazione Internazionale alle attività di ricerca della Sapienza nel Vicino Oriente, in Egitto e nel Mediterraneo.

Il pannello I introduce alle civiltà del Vicino Oriente antico, spiegando quale sia stato il ruolo della Sapienza nella loro riscoperta. Una mappa del Mediterraneo (fig. 2) illustra tutti

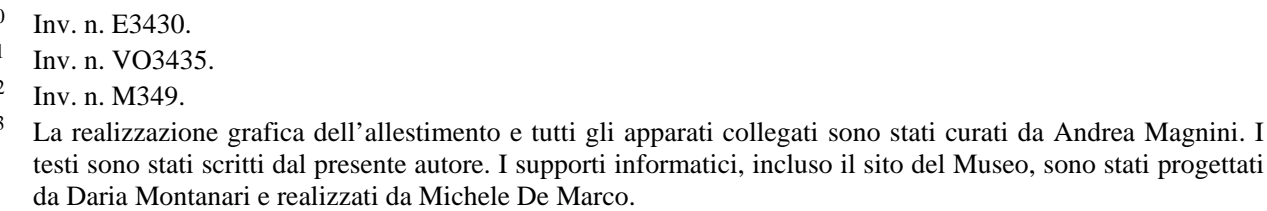


i siti oggetto di ricerche rappresentati nel Museo. Una delle chiavi di lettura offerte al visitatore è quella dello studio del fenomeno urbano nei diversi contesti storici.

Il panello II è dedicato alle conquiste della civiltà neolitica, con un focus particolare su Gerico, e all'invenzione della scrittura cuneiforme in Mesopotamia ${ }^{14}$.

Le vetrine 1-5 sono dedicate a grandi centri del Vicino Oriente nei quali la Sapienza ha lavorato, nell'ordine: Gerico in Palestina (1), Arslantepe in Turchia (2-3), Khirbet alBatrawy in Giordania (4) e Biblo in Libano (5).

Nella vetrina 1 sono esposti reperti che illustrano il periodo Neolitico (su un piano esterno sono due repliche rispettivamente di un mattone "loaf-shaped" del Neolitico Preceramico A e di uno "a sigaro" del Neolitico Preceramico B) ${ }^{15}$, nonché un modello di uno dei teschi modellati del Neolitico Preceramico B (fig. 3), e l'Età del Bronzo, con frammenti ceramici diagnostici e ledge handles, lame di selce, una daga del Bronzo Antico IV, una paletta egiziana in scisto, una testa di mazza in calcare e una giara cananea ${ }^{16}$.

Nella vetrina 2 sono le copie di alcune tra le più significative cretule ritrovate nel palazzo Tardo Uruk di Arslantepe e di alcuni vasi dello stesso periodo illustranti le tecniche di chiusura e controllo dei beni adottate da questa antica istituzione (fig. 4) ${ }^{17}$. Le iconografie mostrano l'emergere di una società complessa basata sull'agricoltura e sul controllo territoriale e commerciale, nella quale emerge una codificata ideologia del potere. Il tema è ripreso in modo altamente esemplificativo dalle straordinarie repliche del corredo di armi e ornamenti appartenuti al principe della successiva città esposte nella vetrina 3 (fig. 5), in una sorta di ricostruzione della tomba scoperta dalla missione diretta da Marcella Frangipane.

La vetrina 4 illustra lo straordinario ritrovamento effettuato nell'antica città giordana di Khirbet al-Batrawy attraverso le repliche delle asce di rame e del vaso cerimoniale ritrovati nella Sala a pilastri del palazzo della metà del III millennio a.C.

Infine, la vetrina 5 , è dedicata a Biblo ${ }^{18}$, un'altra grande capitale del Levante, con immagini del Tempio della Balaat e delle asce d'oro ritrovate in un deposito del Tempio degli Obelischi.

Il pannello III presenta la prima società urbana della Siria Palestina tra III e II millennio a.C., come un unico grande fenomeno storico soffermandosi sui principali esempi scavati dalla Sapienza: Ebla, Gerico e Batrawy. Un cenno importante è fatto alla città di Ugarit, anche per iniziare ad introdurre l'invenzione dell'alfabeto.

Nella vetrina 6 sono esposti reperti provenienti dai corredi tombali della Necropoli di Achziv (oggi in Israele, originariamente in Libano), tra i quali spiccano una figurina fittile di divinità in trono gravida ${ }^{19}$ e diverse brocchette cipro-fenicie in ceramica Red Slip ${ }^{20}$ dipinte con motivi di cerchi concentrici neri e rossi (fig. 6) ${ }^{21}$.

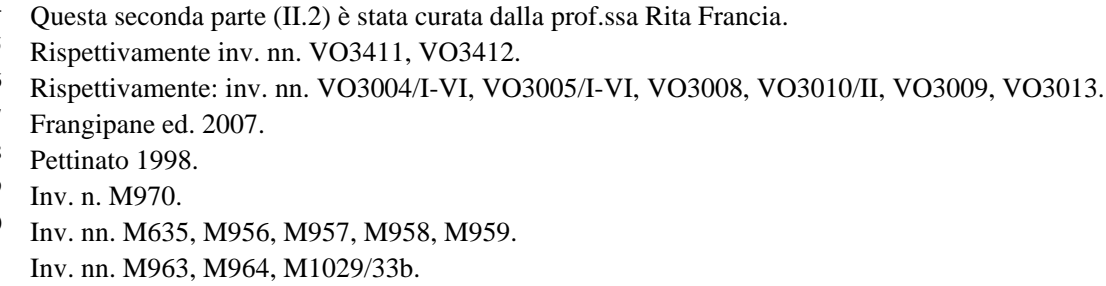


La vetrina 7 contiene alcuni dei numerosi reperti provenienti dallo scavo di Ramat Rahel in Israele (fig. 7). Tra questi sono diverse figurine fittili femminili ${ }^{22}$ (una con mani che sorreggono i seni) ${ }^{23}$, una testa di cavallo fittile ${ }^{24}$, diverse coppe e piatti Red Slip, una brochetta Red Slip, un dipper o attingitoio, un vaso à chardon ${ }^{25}$, due lucerne e due spolette per tessitura in osso $^{26}$.

Sulla pedana 1 , oltre ad un tubo fittile per lo scolo delle acque di età romana ${ }^{27}$, si trovano il frammento di una delle volute di un capitello proto-eolico appartenuto alla facciata del palazzo interno alla Cittadella dell’VIII secolo a.C. e un gesso della ricostruzione completa del capitello (fig. 8) ${ }^{28}$; i calchi degli elementi architettonici con corone di petali rivolti in basso che venivano utilizzati come balaustre nelle finestre o nei balconi ${ }^{29}$, appartenuti sempre allo stesso complesso monumentale del palazzo di Ramat Rahel.

Il pannello IV è posizionato sulla pedana 1, dedicata alla esposizione degli elementi architettonici appartenuti al palazzo reale scavato a Khirbet Salih, l'antica Ramat Rahel. Esso descrive la Cittadella dei re di Giuda a Ramat Rahel dei secoli VIII-VII a.C. e, sulla pedana 2, i ritrovamenti relativi alla fase bizantina.

Sulla pedana 2, posizionata accanto alla vetrina 7, che raggruppa anch'essa materiali da Ramat Rahel, sono alcune anfore e un grande bacino dell'VIII-VII secolo a.C. ${ }^{30}$, un ossuario romano del I secolo d.C. con iscrizione ( $\Sigma \mathrm{IM} \Omega \mathrm{NI} \Delta \mathrm{H} \Sigma$ ) (fig. 9) ${ }^{31}$, un'anfora bizantina a pareti corrugate e un'anfora islamica con decorazione pettinata ${ }^{32}$. Questi ultimi reperti aprono la sezione dedicata a Ramat Rahel post-israelita, dopo la conquista romana nel 64 d.C. e la completa trasformazione ad opera della $X$ Legio del sito in un'ampia azienda agricola, che, con diverse vicissitudini, rimase in uso fino a tutta l'epoca islamica.

La vetrina 8 contiene reperti di epoca ellenistica e romana da Khirbet Salih (Ramat Rahel): oltre ad alcuni laterizi con lo stampiglio della $X$ Legio Fretensis ${ }^{33}$, diverse monete $^{34}$ (una di Agrippa del 43-42 a.C.) ${ }^{35}$, alcune lucerne ${ }^{36}$, un punta di freccia d'età erodiana ${ }^{37}$, il piede di una figurina in terracotta ${ }^{38}$, un anello di bronzo ${ }^{39}$ e una bottiglietta

22 Inv. nn. M104, M126, M256 (Ciasca 1960, 23, tavv. 10-11).

23 Inv. n. M55 (Aharoni 1964, tav. 36:1).

24 Inv. n. M53.

25 Rispettivamente: inv. nn. M148, M176, M105, M185, M127 (Aharoni 1964, 29-32, fig. 18:22, tavv. 32:2, 33).

26 Inv. nn. M203, M966, M232, M157.

27 Inv. nn. M64-67 (Ciasca 1960, 30, tav. 16).

28 Rispettivamente: inv. nn. M70, M93 (Ciasca 1960, 21, tavv. 6, 9; Aharoni 1964, 28-29, tav. 42).

29 Inv. n. M209 (Ciasca 1960, 22-23; Aharoni 1964, 56-58, tavv. 44:2, 45-48).

30 Inv. nn. M185, M186, M187, M196, M684 (Aharoni 1964, 30-31, tav. 32:1, 3).

31 Inv. nn. M92, M255 (Kochavi 1964, 70-73, fig. 29, tav. 10).

32 Rispettivamente: inv. nn. M20, M490.

33 Inv. n. M74/I-II (Ciasca 1960, 31, tavv. 20-21).

34 Inv. nn. M61/4, 5, 9, 10, 11, 12 (Ciasca 1960, 31; Rahmani 1964, 107-117).

35 Inv. n. M61/3.

36 Inv. nn. M77, M201, M300, M307.

37 Inv. n. M233.

38 Inv. n. M266 (Aharoni 1964, 42, tav. 8:4).

39 Inv. n. M253. 
di vetro del III secolo d.C. ${ }^{40}$ Essa testimonia, anche attraverso il pannello interno, il radicale cambiamento imposto alla cittadella israelita, distrutta dai Romani e trasformata in una fiorente fattoria.

La vetrina 9 illustra la lunga storia di Khirbet Salih in epoca bizantina e islamica, quando il sito fu centro di produzione di olio e di vino, come mostrano le cinque anfore corrugate disposte fuori della vetrina stessa lungo le pareti della sala ${ }^{41}$. Oltre ad un'anfora bizantina e a diverse pentole, nella vetrina è una collezione di lucerne (fig. 10) e alcuni frammenti di ceramica invetriata ${ }^{42}$. I materiali datano tra il IV e l’VIII secolo d.C.

Il roll-up sull'alfabeto introduce, subito dopo la vetrina 9 che chiude l'ampia sezione espositiva dedicata a Khirbet Salih/Ramat Rahel, il nuovo ambito tematico individuato dal colore azzurro, ossia l'espansione dei Fenici verso Occidente e la formazione della civiltà mediterranea tra la fine del II e gli inizi del I millennio a.C. I più recenti ritrovamenti che consentono di rintracciare le origini levantine dell'alfabeto e una tavola comparativa delle scritture illustrano quello che fu lo strumento chiave per la nascita della civiltà mediterranea.

I pannelli V e VI trattano rispettivamente l'espansione fenicia in Occidente attraverso le rotte che condussero i Fenici oltre le Colonne d'Ercole nell'Atlantico sino a Cadice e Lixus, la presenza fenicia a Cipro e l'emergere di Cartagine come centro egemone del Mediterraneo centrale tra VI e II secolo a.C.

La vetrina 10 è dedicata a Malta, dove la Sapienza è attiva dal $1963^{43}$ e dove fondamentale è stato l'impegno nello scavo del grande santuario di Astarte (successivamente Hera e Giunone) a Tas Silg, impiantato dai Fenici sulle vestigia del grande tempio megalitico preistorico di Marsalokk ${ }^{44}$. Lo studio di più di 8000 iscrizioni dedicatorie rinvenute in questo tempio, le ricerche condotto anche a San Pawl Milqi e, nell'isola di Gozo, a Ras al-Wardija e, infine, il recente ritrovamento di un pendente in agata con un'iscrizione cuneiforme paleobabilonese ${ }^{45}$, sono tra i più importanti risultati delle ricerche della Sapienza illustrati dalla vetrina ${ }^{46}$.

Nella vetrina 11 (fig. 11) sono raccolti esemplificativamente reperti provenienti da Cipro (Kition), da Pantelleria (di grande interesse la caratteristica ossidiana pantesca), dalla Tunisia dove la Sapienza condusse fondamentali ricerche sul Capo Bon, documentando la serie di fortezze che controllavano il Canale di Sicilia e proteggevano il Golfo di Cartagine (in particolare scavando quella di Ras ed-Drek), dall'Algeria (Banasa) e, infine, dal Marocco (Mogador), testimoniando l'ampiezza delle ricerche condotte seguendo le diverse mete dell'espansione fenicia.

40 Inv. n. M251 (Aharoni 1964, 40, tav. 6:3).

41 Inv. nn. M14, M93, M240, M242, M3859/1.

42 Rispettivamente: inv. nn. M62, M239, M418, M3849/3, M33, M36, M41, M77, M146, M163, M169, M177, M178, M179, M183, M195, M196, M197, M204, M205, M243, M244, M245, M312, M118/50, M1476/1 (Ciasca 1960, 34, tavv. 31, 32).

43 Ciasca - Rossignani 1998

44 Ciasca 1976-1977; Amadasi Guzzo 2011.

45 Cazzella - Pace - Recchia 2011.

46 Inv. nn. M547, M658, M661, M930, M931, M 933, M1014, M1124, M1239, M1148 (Amadasi Guzzo 2011, 21, fig. 13). 
Al lato della vetrina 11 la testa di una statua schematica proveniente da Malta datata al III secolo a.C. (fig. 12) ${ }^{47}$. Al di sotto, inizia il partito espositivo della vetrina 12 con un pannello che illustra le scoperte della Missione archeologica a Mozia con particolare focus sul primo insediamento dei Fenici nell'isola. Un'àncora arcaica è esposta al di sotto di questo pannello. Nella vetrina adiacente sono il corredo di una tomba del VII secolo a.C., costituito da un'olla, una brocchetta e un grande piatto dipinti ${ }^{48}$; due matrici in terracotta, una per la produzione di una protome di Demetra e l'altro per stampigliare presumibilmente il lato principale di un'arula con la raffigurazione di una sfinge che divora un personaggio maschile ${ }^{49}$; un falcetto in ferro ritrovato nel Santuario delle Acque del V secolo a.C. ${ }^{50}$, due arule decorate a stampo, una con il tema dei due grifi che atterrano un equide ${ }^{51}$ e una con una sfinge alata tra due stendardi sormontati da palmette ${ }^{52}$.

Di fronte alla vetrina 12 si trova la vetrina 13, con la colonnina scanalata sormontata da un capitello eolico rinvenuta nella "Casa del sacello domestico" ${ }^{53}$ e, a fianco, una pedana sostiene il grande pithos dipinto ritrovato a Mozia nella Fortezza Occidentale ${ }^{54}$.

Un'ampia installazione è realizzata sulla pedana 3, sormontata da una grande foto aerea di Mozia, che ospita nella metà sinistra una semplice ricostruzione del campo di urne e di stele del Tofet di Mozia ${ }^{55}$, corredata di stele ${ }^{56}$ e di urne e, in quella destra, una ricostruzione del Tofet di Monte Sirai, in Sardegna, anch'essa con stele ${ }^{57}$, un'urna ${ }^{58}$, due piccole anfore ${ }^{59}$, e anche una grande anfora ${ }^{60}$ (fig. 13). Al centro, in uno spazio libero, sono alcuni strumenti dell'archeologo: la piccozzina, la trowel, il filo a piombo, il metro a stecca e una caldarella.

La successiva vetrina 14 , che delimita la pedana 3 a destra, è dedicata a Monte Sirai, sito scoperto dalla Sapienza, di cui il MVOEM conserva diversi corredi tombali ${ }^{61}$ con brocchette Red Slip ${ }^{62}$, coppe, piatti ${ }^{63}$, avori (inclusa la famosa palmetta che è divenuta il simbolo del Museo, fig. 14) ${ }^{64}$, e alcuni importanti reperti dal

Inv. n. M661.

48 Rispettivamente inv. nn. M3451, M3452, M3453.

49 Rispettivamente: inv. nn. M3454, M3455 (Nigro 2015a, fig. 9).

Inv. n. M3456.

Inv. n. M3457 (Nigro 2011, 98-99, fig. 3.95, tav. IV).

Inv. n. M3458 (Nigro 2012, 210, fig. 32.2).

Inv. n. M3459 (Nigro 2001-2003).

Inv. n. M3460 (Nigro 2004-2005).

Ciasca 1992; Nigro 2012; 2013.

Inv. nn. M577 (calco; Forte 1966, 84-85, n. 55, tav. LXV), M576 (calco; Forte 1966, 78, n. 47).

57 Inv. nn. M352 (Garbini 1964, 79, n. 10, tav. XL), M354 (Garbini 1964, 81, n. 14, tav. XXXVI), M358 (Garbini 1964, 93, n. 53, tav. XLV).

58 Urna e piattelo: inv. nn. M362, M361.

59 Inv. nn. M346, M374.

60 Inv. n. M94 (Barreca 1964, 47 n. 2/2, tav. 29, destra).

61 Barreca 1964, 36-55; Amadasi - Brancoli, 1965; Fantar - Fantar 1966; Bartoloni 2000.

62 Inv. nn. M385, M387, M389, M488, M503, M505.

63 Inv. nn. M336, MMSN/82, MMSN/252, M88, M362/2, M484, M485.

64 Inv. n. M921 (Barreca 1965, 56, tav. XXXI:1). 
Tofet ${ }^{65}$, tra i quali una statuetta in terracotta del dio Baal Hammon itifallico ${ }^{66}$, una di divinità assisa in trono ${ }^{67}$ ed una laminetta bronzea iscritta con una dedica ad Astarte ${ }^{68}$. A fianco della vetrina è il pannello VII che illustra le testimonianze e le peculiarità della presenza dei Fenici in Sardegna, ricordando anche l'iniziale impegno della Sapienza a Sulky e a Tharros.

Infine, la Sezione Vicino Oriente e Mediterraneo termina con la vetrina 15, destinata alle esposizioni temporanee, per il momento dedicata alle attività delle Missioni in Iraq, a Ur, Abu Tbeirah e Eridu.

La Sezione Egitto si dispiega nella metà destra della sala piacentiniana e si apre con il pannello XI che ospita una grande mappa dell'Egitto antico e la cronologia completa dei Faraoni. Davanti al pannello e addossata ad uno dei pilastri d’ingresso è la copia in gesso del busto della statua della regina Tuya (fig. 15) ${ }^{69}$, madre di Ramses II, il cui originale di granito si trova oggi ai Musei Vaticani (al pilastro opposto, è invece addossata una semplice tavola cronologica del Mediterraneo dal Neolitico al Novecento). In fondo, sulla parete della sala, è la replica di una testa non finita della regina Nefertiti, moglie di Akhenaton, ritrovata nella bottega dello scultore Thutmosis a el-Amarna. Quest’opera è un dono del Prof. Alfonso Archi.

La prima vetrina della Sezione Egitto è la 16 e raccoglie alcune opere della vita quotidiana: una statuetta di legno ${ }^{70}$, una statuina della dea Thoeri ${ }^{71}$, un pezzo di corda, scarabei, terrecotte, figurine di ushabti, ornamenti ${ }^{72}$. La vetrina 17 è invece dedicata all'immagine del faraone e conserva tre opere di valore: una testa mutila in quarzite di un faraone della XII o XIII dinastia ${ }^{73}$, una testa di una statua lignea intonacata di un faraone della XVIII dinastia ${ }^{74}$ (una statua di $k a$, forse di Amenofis II), una terza testa sempre di legno non identificabile ${ }^{75}$. La vetrina 18 come anche parte della adiacente pedana 4 sono dedicate alla statuaria comune con esempi di statue di funzionari di varia epoca ${ }^{76}$.

La stessa pedana (fig. 16) è sormontata sulla parete da diverse copie dei rilievi e delle iscrizioni geroglifiche ${ }^{77}$ della tomba di Sheshonq (TT 27), un alto funzionario vissuto durante la XXVI dinastia (fig. 17), la cui tomba monumentale nella necropoli di Asasif a

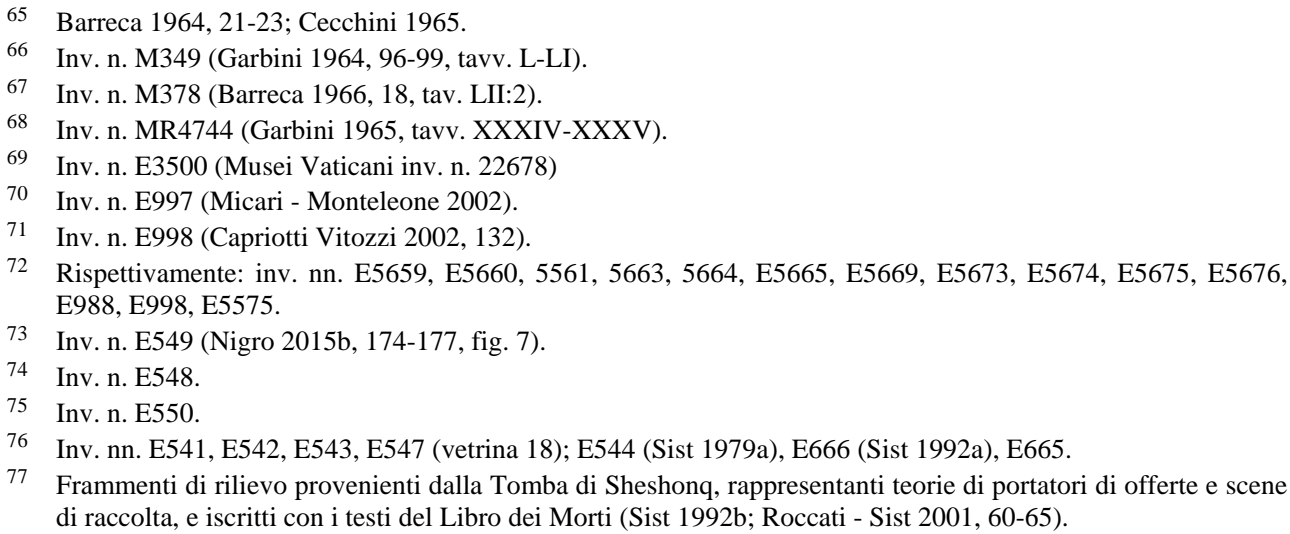


Tebe è stata esplorata e in parte restaurata dalla Sapienza tra 1969 e $2008^{78}$. Più in alto sopra la pedana sono due lati di un sarcofago ligneo dipinto della stessa dinastia, appartenuto tuttavia ad un personaggio di rango assai minore ${ }^{79}$. La pedana 4 ospita poi numeroso vasellame appartenuto a tombe predinastiche scavate rispettivamente ad Antinoe ${ }^{80}$ e Tamit $^{81}$ e, all'estremità destra, una copia in gesso della sfinge del regno di Thutmosis III oggi al Museo Barracco a Roma ${ }^{82}$. Altri corredi dalle stesse necropoli, contraddistinti dalla presenza di vasi ad alette, pietre preziose, palette, che mostrano l'asserzione del rango nelle prime élites collegate al potere reale nell'Egitto predinastico, sono esposti nelle vetrine $19^{83}$ e 20 (fig. 18) ${ }^{84}$. Infine, la vetrina 21, presenta alcuni interessanti reperti dalla stessa tomba di Sheshonq ${ }^{85}$.

Con il pannello VIII si illustra lo straordinario sforzo compiuto dalla missione in Egitto e Nubia diretta da Sergio Donadoni tra 1964 e 1970. Negli anni 1965-1968 furono condotte quattro campagne ad Antinoe ${ }^{86}$, mentre nel 1964 una campagna di salvataggio nel sito di Tamit ${ }^{87}$, una cittadina paleocristiana in Nubia cui sono dedicate le vetrine 22 e 23, con reperti che coprono le tre fasi maggiori dell'insediamento tra VIII e XII secolo d.C. ${ }^{88}$ In entrambi i luoghi furono anche identificate due necropoli predinastiche, che hanno restituito cospicui corredi, visibili nelle vetrine 19 e 20. La seconda parte del pannello VIII è invece dedicata alla straordinaria impresa del salvataggio della Chiesa di Sonqi Tino ${ }^{89}$, i cui dipinti parietali ${ }^{90}$, rarissima testimonianza dell'arte paleocristiana copta, sono esposti nelle sale del Palazzo del Rettorato adiacenti il Museo VOEM. Sotto al pannello è la pedana 5 (fig. 19), sulla quale sono disposti numerosi reperti, vasi e incensieri a rocchetto trovati nella chiesa, incluse due pietre di fondazione contenute originariamente all'interno dell'altare in mattoni crudi del haikal. Una mensola aggettante dallo stesso pannello VIII sostiene lo straordinario calice in ceramica arancione con labbro nero ritrovato nella chiesa ${ }^{91}$ (fig. 19).

78 Donadoni 1973; 1976; 1993; Contardi 2010

79 Inv. n. E1000 (Sist 2013)

80 Corredi vascolari delle Tombe 2, 5, 6: vasi cilindrici a bocca anulare e prese ondulate laterali (inv. nn. E1008, E1009, E1010, E1028, E1032), vasi cilindrici a bocca anulare con piccola fascia incisa sotto il bordo (inv. nn. E1035, E1037, E1039, E1040), giare ovoidali con orlo arrotolato, breve collo, fondo a punta arrotondata (inv. nn. E41, E44, E1003, E1006, E1012, E1013, E1029, E1030, E1031; Spallanzani Zimmermann 1974).

81 Corredi vascolari delle Tombe 9 e 11: coppa con orlo piatto (inv. n. E1680); piatti con l'orlo lievemente arrotondato, interno nero, esterno rosso, orlo nero, incisioni verticali sul corpo e diagonali sull'orlo (inv. nn. E28, E1678); vasi con orlo piatto, fondo a punta, interno nero, esterno rosso, orlo nero, incisioni verticali sul corpo, diagonali sull'orlo (inv. nn. E23, E27, E43, E71, E1679, E1681, E1684; Bosticco 1967a, 88-94). Inv. n. E3440 (Museo Barracco inv. n. MB13).

83 Inv. nn. E1001, E1002, E1007, E1007bis, E1019, E1056, E1027, E1016, E1017, E1018, E1020, E1023, E1026, E1033, E1034, E1035, E1038, E1042, E1043, E1047, E1048, E1054, E1057.

84 Inv. nn. E1, E2, E3, E4, E5, 6, 7, 42, E58, E60, E65, E66, E67, E68, E996, E1656, E1659, E1168, E1669, E1676.

85 Inv. nn. ET27/3, ET72/26, ET72/46, ET72/51, ET72/54.

86 Donadoni 1974

87 Bosticco 1967b.

88 Inv. nn. E109, E118, E129, E135, E146, E152, E196, E293, E1660 (Roveri 1967).

89 Fanfoni (a cura di) 1979; Sist 2012.

90 Pasi 2012.

91 Inv. n. E1638 (Angelozzi - Iob 2012, 627, fig. 5). 
Sulla parete accanto al pannello sono alcuni frammenti di decorazione scultorea ${ }^{92}$ dallo stesso monumento e un affresco della Vergine con Gesù bambino raffigurati su un motivo stilizzato di montagna ${ }^{93}$ (X secolo d.C.), il graffito con il nome del possibile santo titolare della chiesa: MIXALH, che appare anche in un rilievo ${ }^{94}$ (fig. 19).

Il pannello IX ha mere funzioni didattiche e illustra la scrittura geroglifica, le piramidi e il regno dei morti nell'Egitto faraonico.

Una stele copta ${ }^{95}$, tre anfore Dressel II- $4^{96}$, un mortaio da Antinoe ${ }^{97}$ e il calco di un capitello da Ramat Rahel ${ }^{98}$, sono disposti lungo la parete terminale di questa sezione del Museo, che ospita un grande touch-screen e le installazioni per le attività didattiche con le scuole.

Con il pannello $\mathrm{X}$, riprende il percorso espositivo con un'ampia presentazione dell'Egitto ellenistico e di quello romano, con Antinoupoli la città fondata da Adriano nel 131 d.C. in memoria del proprio favorito, divenuta uno dei più fiorenti crocevia commerciali e culturali dell'Egitto imperiale. Le vetrine 24 e 25 (fig. 20) illustrano con numerosi variegati reperti, la congerie culturale di questo centro, con notevoli esempi di ceramica figurata e figurine dipinte (vetrina 24) ${ }^{99}$, frammenti di tessuti di lino dipinti, avori e ornamenti (vetrina 25) ${ }^{100}$.

All'epoca tardo-ellenistica e romana appartengono anche gli straordinari reperti provenienti dal Palazzo del sovrano Natakamani a Napata (I secolo d.C.) ${ }^{101}$, in Nubia, presso il Jebel Barkal. Si tratta di ceramiche variopinte e di frammenti di decorazioni architettoniche come stucchi invetriati esposti nella vetrina $26^{102}$. Sull'adiacente pedana 6 trovano posto il calco di un leone (fig. 21) ${ }^{103}$ posto a guardia di uno degli ingressi del palazzo e quello di una stele iscritta in meroitico rinvenuta nel $1984^{104}$. Sulla parete sopra la pedana è appeso un plastico che raffigura le fondazioni del palazzo portato alla luce dalla missione della Sapienza al Jebel Barkal, in Sudan.

La successiva vetrina 27 è invece dedicata a vasellame, figurine in terracotta, lucerne e ornamenti e oggetti di bronzo provenienti da Arsinoe, nel Fayyum ${ }^{105}$. I materiali risalgono

92 Inv. nn. E104, E343, E345.

93 Pasi 2012, 576-577, fig. 10

94 Inv. n. E2011.

95 Inv. n. E131 (Donadoni 1967, 72-73).

96 Inv. nn. ES713-ES715.

97 Inv. n. EC67.52.

98 Inv. n. M318 (Ciasca 1960, 22, tav. 7)

99 Inv. nn. E999, E1221, E1467-E1468, E1472-E1473, E1480, E1485, E1598, E1600, E1601-E1604, E1606 E1608, E1610-E1616, E1630, E1632-E1633 (Guerrini 1974; Sist 1996).

100 Inv. nn. ES533, ES534, E1114, E1120-E1121, E1129-E1130, E1132, E1138, E1158-E1176, E1179, E1181E1191, E1193-E1196, E1200, E1202, E1209-E1210, E1315, E1321, E1328, E1335-E1336, E1346, E1358, E1363, E1369, E1370-E1373.

101 Sist 2000; 2005; 2011.

102 Inv. nn. E115, E233, E913, E1852, E1856, E1867-E1869, E1877-E1879, E1884, E1891, E1896, E1904, E1906-E1907, E1910, E1915, E1917, E1919, E1909, EGB74G7, E2014 (Sist 1982).

103 Inv. n. E3413.

104 Inv. n. E3414 (Tiradritti 1992).

105 Inv. nn. E1152, E1154, E1156, E1319-E1320, E1359, E1425, E1427, E1430-E1432, E1436, E1517, E1573, E1580-E1585, E1587-E1590, E1319-E1320. 
a epoche diverse dall'età ellenistica a quella cristiana, come illustra un'iscrizione copta su un'anfora ${ }^{106}$.

La vetrina 28 è dedicata all'esposizione temporanea e, al momento, contiene tre stele iscritte di varia data (una del Primo Periodo Intermedio, una d'età tolemaica e una d'età romana, nonché un papiro con un testo in aramaico datato al II secolo a.C.) ${ }^{107}$ (fig. 22).

Infine, la vetrina 29, addossata al lato destro del pilastro d'ingresso, è riservata allo straordinario piatto in pietra tufacea giallo-ocra che reca, sotto l'orlo l'iscrizione del nome di Hotepsekhemwy (fig. 23), primo faraone della II Dinastia ${ }^{108}$. Questo reperto è, senza dubbio, il più prezioso nella Sezione Egitto e fotografa un momento cruciale della storia dell'antico Egitto.

I due itinerari delle Sezioni Vicino Oriente - Mediterraneo ed Egitto sono semplicemente suggeriti al visitatore che è libero di seguire un proprio percorso di scoperta del Museo VOEM e dei suoi numerosi reperti. I contenuti dei pannelli esplicativi e le didascalie dei pezzi sono disponibili online e nel Museo è attivo un sistema wifi ad accesso libero per consentire ai visitatori di collegarsi e poter leggere, attraverso il QR code stampato accanto ai numeri delle vetrine, tutti gli apparati illustrativi.

Alcune opere sono collocate al di fuori della sala piacientiniana. In particolare gli affreschi di Sonqi Tino sono esposti nella galleria della terrazza posteriore del Palazzo del Rettorato (fig. 24), dove è stato anche collocata la copia in gesso del monumentale rilievo del Tempio del dio leone a Mussawwarat es-Sufra in Nubia (Sudan settentrionale).

\section{ATtIVITÀ DEL MUSEO VOEM E SUO RUOLO OGGI}

Il Museo VOEM è una struttura di ricerca che, allo stesso tempo, persegue la terza missione dell'Università, quella della dissemination, la divulgazione e formazione scientifica rivolta al pubblico generalista e, in particolare, ai bambini. Mentre si è dato il via ad una completa revisione inventariale, concentrata specialmente sui vasti corpora di materiali non in esposizione, che è accompagnata da documentazione grafica e fotografica, analisi e restauro, allo stesso tempo sono state realizzate diverse iniziative come incontri, seminari, conferenze, concerti atti a rendere vivo il Museo come uno spazio di riflessione e di conoscenza delle culture antiche del Vicino Oriente, dell'Egitto e del Mediterraneo (si veda il Bollettino in questo volume), specialmente in questo tragicissimo momento storico, nel quale proprio il patrimonio archeologico di questa area del mondo è oggetto di un attacco violento ed efferato privo di precedenti. Il Museo si propone anche di conservare e tramandare la documentazione degli scavi i cui risultati sono in esso rappresentati e quindi di testimoniare il grande sforzo compiuto dalla Sapienza negli ultimi cinquant'anni con l'aiuto prezioso e autorevole del Ministero degli Affari Esteri alla base di tanti positivi rapporti costruiti nei diversi Paesi con la popolazione locale e le Autorità preposte alla tutela e alla valorizzazione dei monumenti e dei beni archeologici.

106 Inv. n. E1574.

107 Rispettivamente: inv. nn. ES709 (Roccati 1985); E1699 (Sist 1979b); E1688 (Donadoni 1986).

108 Inv. n. E2106 (Sist 2014; Nigro 2015b, 170-174). 


\section{BIBLIOGRAFIA}

AHARONI, Y.

1964 Excavations at Ramat Rahel. Seasons 1961 and 1962 (Centro di Studi Semitici. Serie archeologica 6), Roma 1964.

AmADASI GUZZO, M.G.

2011 Missione Archeologica Italiana a Malta. Il santuario di Astarte di Malta: le iscrizioni in fenicio da Tas-Silg, Roma 2011.

AMADASI, M.G. - BRANCOLI, I.

1965 La necropoli: M.G. AMADASI - F. BARRECA - P. BARTOLONI - I. BRANCOLI - S.M. CECCHINI - G. Garbini - S. Moscati - G. Pesce, Monte Sirai - II. Rapporto preliminare della Missione archeologica dell'Università di Roma e della Soprintendenza alle Antichità di Cagliari (Studi Semitici 14), Roma 1965, pp. 95-121.

ANGELOZZI, M. - IoB, A.

2012 La cultura materiale di Sonqi Tino: Scienze dell'Antichità 18 (2012), pp. 625-637.

BARRECA, F.

1964 Gli scavi: F. BARRECA - G. GARBINI, Monte Sirai - I. Rapporto preliminare della Missione archeologica dell’Università di Roma e della Soprintendenza alle Antichità di Cagliari (Studi Semitici 11), Roma 1964, pp. 11-63.

1965 Confronti e conclusioni: M.G. AMADASI - F. BARRECA - P. BARTOLONI - I. BRANCOLI S.M. Cecchini - G. Garbini - S. Moscati - G. Pesce, Monte Sirai - II. Rapporto preliminare della Missione archeologica dell'Università di Roma e della Soprintendenza alle Antichità di Cagliari (Studi Semitici 14), Roma 1965, pp. 19-62.

1966 Il Mastio: M.G. AMAdASI - F. BARRECA - G. GARBINI - M. FANTAR - D. FANTAR - S SORDA, Monte Sirai - III. Rapporto preliminare della Missione archeologica dell’Università di Roma e della Soprintendenza alle Antichità di Cagliari (Studi Semitici BARTOLONI, P. 20), Roma 1966, pp. 9-54.

2000 La necropoli di Monte Sirai - I (Collezione di Studi Fenici 41), Roma 2000.

Bosticco, S.

1967a Le necropoli: Tamit (1964). Missione Archeologica in Egitto dell'Università di Roma (Istituto di Studi del Vicino Oriente. Serie Archeologica 14), Roma 1967, pp. 85-94.

1967b Topografia e urbanistica: Tamit (1964). Missione Archeologica in Egitto dell'Università di Roma (Istituto di Studi del Vicino Oriente. Serie Archeologica 14), Roma 1967, pp. 17 26.

CAPRIOTTI ViTOZZI, G.

2002 Thoeri lactans: A. RocCATI - G. CAPRIOTTI VITTOZZI (a cura di), Tra le palme del Piceno: Egitto terra del Nilo, Poggibonsi 2002, p. 132.

CAZZella, A. - PACE, A. - ReCCHIA, G.

2011 Dal santuario di Tas-Silg a Malta un'iscrizione cuneiforme del II millennio a.C.: culti e scambi nel Mediterraneo: Scienze dell’Antichità 17 (2011), pp. 599-609.

CECCHINI, S.M.

1965 Il «Tophet»: M.G. AMADASI - F. BARRECA - P. BARTOLONI - I. BRANCOLI - S.M. CECCHINI G. Garbini - S. Moscati - G. Pesce, Monte Sirai - II. Rapporto preliminare della Missione archeologica dell'Università di Roma e della Soprintendenza alle Antichità di Ciasca, A. Cagliari (Studi Semitici 14), Roma 1965, pp. 123-133.

1960 I ritrovamenti: S. MosCATI, Il Colle di Rachele (Ramat Rahel) (Centro di Studi Semitici e dell’Antico Oriente), Roma 1960, pp. 15-37. 
1976-1977 Il tempio fenicio di Tas-Silg: una proposta di ricostruzione: Kokalos 22-23 (1976-1977), pp. 162-172.

1992 Mozia: sguardo d'insieme sul Tofet: Vicino Oriente VIII (1992), pp. 113-155.

Ciasca, A. - MatThiae, P. - Sist, L.

1993 Museo del Vicino Oriente: M. Barbanera - I. Venafro (a cura di), I Musei dell’Università «La Sapienza», Roma 1993, pp. 261-276.

CiAsCA, A. - ROSSIGNANI, M.P.

1998 Malta: L. Drago Troccoli (a cura di), Scavi e ricerche archeologiche dell'Università di Roma “La Sapienza” (Studia Archeologica 96), Roma 1998, pp. 233-237.

CONTARDI, F.

2010 La tomba di Sheshonq (TT 27) all’Asasif. Missione Archeologica dell’Università di Roma "La Sapienza”. Campagne 2005, 2008: R. PIRELli (a cura di), Ricerche italiane e scavi in Egitto IV, Il Cairo 2010, pp. 105-110.

DONADONI, S.

1967 Le iscrizioni: Tamit (1964). Missione Archeologica in Egitto dell'Università di Roma (Istituto di Studi del Vicino Oriente. Serie Archeologica 14), Roma 1967, pp. 61-74.

1973 Relazione preliminare di scavo sulla II campagna di scavo nella tomba di Šešonq all'Asasif (1971): Oriens Antiquus 12 (1973), pp. 19-22.

1974 Introduzione: Antinoe (1965-1968). Missione Archeologica in Egitto dell'Università di Roma (Istituto del Vicino Oriente. Serie Archeologica 21), Roma 1974, pp. 15-21.

1976 L’inquadramento archeologico: Oriens Antiquus 15 (1976), pp. 209-217.

1983 Una testata di decreto tolemaico: N. BonACASA - A. Di ViTA (a cura di), Alessandria e il mondo ellenistico-romano: studi in onore di Achille Adriani (Studi e Materiali. Istituto di Archeologia di Palermo 6), Roma 1983, pp. 162-164.

1993 La situazione archeologica: Vicino Oriente IX (1993), pp. 7-14.

FANFONI, G. (a cura di)

1979 Sonqi Tino I. L'architettura della chiesa (Istituto di Studi del Vicino Oriente. Serie Archeologica 24), Roma 1979.

FANTAR, M. - FANTAR, D.

1966 La nécropole: M.G. AMADASI - F. BARRECA - G. GARBINI - M. FANTAR - D. FANTAR - S. SORDA, Monte Sirai - III. Rapporto preliminare della Missione archeologica dell'Università di Roma e della Soprintendenza alle Antichità di Cagliari (Studi Semitici 20), Roma 1966, pp. 63-81.

FORTE, M.

1966 Catalogo delle stele: A. CiAsCA - M. ForTE - G. GARBINI - V. TUSA - A. TUSA CUTRONI - A VERGER, Mozia - II. Rapporto preliminare della Missione archeologica della Soprintendenza alle Antichità della Sicilia occidentale e dell'Università di Roma (Studi Semitici 19), Roma 1966, pp. 71-108.

FRANGIPANE, $\mathrm{M}$.

1998 Arslantepe-Malatya: L. Drago Troccoli (a cura di), Scavi e ricerche archeologiche dell’Università di Roma “La Sapienza” (Studia Archeologica 96), Roma 1998, pp. 330340.

FrangIPANE, M. (ed.)

2007 Arslantepe Cretulae. An Early Centralised Administrative System Before Writing (Missione Archeologica Italiana nell’Anatolia Orientale, Arslantepe V), Rome 2007.

GARBINI, G.

1964 I monumenti figurati: F. BARRECA - G. GARBINI, Monte Sirai - I. Rapporto preliminare della Missione archeologica dell'Università di Roma e della Soprintendenza alle Antichità di Cagliari (Studi Semitici 11), Roma 1964, pp. 65-99. 
1965 L'iscrizione punica: M.G. AMADASI - F. BARRECA - P. BARTOLONI - I. BRANCOLI - S.M. CECChini - G. GARBini - S. MosCATI - G. Pesce, Monte Sirai - II. Rapporto preliminare della Missione archeologica dell'Università di Roma e della Soprintendenza alle Antichità di Cagliari (Studi Semitici 14), Roma 1965, pp. 79-92.

GUERRINI, L.

1974 Materiali ceramici: Antinoe (1965-1968). Missione Archeologica in Egitto dell'Università di Roma (Istituto del Vicino Oriente. Serie Archeologica 21), Roma 1974, pp. 69-113.

MicARi, C. - MonTELEONE, M.

2002 Statuetta lignea: A. RocCATI - G. CAPRIOTTI VitTOZZI (a cura di), Tra le palme del Piceno. NigRo, L. Egitto terra del Nilo, Poggibonsi 2002, pp. 156-157.

2001-2003 Un arredo in terracotta conformato a capitello eolico da Mozia: Scienze dell'Antichità 11 (2001-2003), pp. 567-577.

2004-2005 Un pithos dipinto dalla Fortezza Occidentale di Mozia: Scienze dell'Antichità 12 (20042005), pp. 727-737

2011 Mozia - XIII. Zona F. La Porta Ovest e la Fortezza Occidentale. Rapporto preliminare. delle campagne di scavi XXIII-XXVII (2003-2007) condotte congiuntamente con il Servizio Beni Archeologici della Soprintendenza Regionale per i Beni Culturali $e$ Ambientali di Trapani (Quaderni di Archeologia Fenicio-Punica, VI), Roma 2011.

2012 Scavi e restauri dell’Università di Roma 'La Sapienza' a Mozia, 2007-2009: il Tempio del Kothon, il Temenos Circolare, il Sacello di Astarte e il Tofet: C. Ampolo (ed.), Sicilia occidentale. Studi, rassegne, ricerche. Atti delle settime giornate di studi sull'area elima e la Sicilia occidentale nel contesto mediterraneo, Erice 12-15 ottobre 2009 (Seminari e convegni 29), Pisa 2012, pp. 207-218.

2013 Mozia: il Tofet e la città. Il limite meridionale del santuario e le strutture collegate negli scavi della Sapienza 2010-2011: Scienze dell'Antichità 19 (2013), pp. 37-53.

2015a Mozia tra VI e V secolo a.C. Monumentalizzazione e organizzazione socio-politica: un nuovo modello: M.P. BAGLIONE - L.M. Michetti (a cura di), Le lamine d'oro a cinquant'anni dalla scoperta. Dati archeologici su Pyrgi e rapporti con altre realtà del Mediterraneo (Scienze dell'Antichità 21.2), Roma 2015, pp. 225-245.

2015b Memoria conservata e memoria violata: M. BARBANERA - A. CAPODIFERRo (a cura di), La Forza delle Rovine, Roma 2015, pp. 168-177.

PASI, S.

2012 I dipinti della chiesa di Sonqi Tino in Nubia: Scienze dell'Antichità 18 (2012), pp. 569591.

Pettinato, G.

1998 Missione archeologica italiana a Byblos: L. DRAGo Troccoli (a cura di), Scavi e ricerche archeologiche dell’Università di Roma “La Sapienza” (Studia Archeologica 96), Roma 1998, pp. 313-315

RAHMANI, L.Y.

1964 Description of the coins: Y. AHARONI, Excavations at Ramat Rahel. Seasons 1961 and 1962 (Centro di Studi Semitici. Serie archeologica 6), Roma 1964, pp. 107-117.

ROCCATI, A.

1985 La stele di un falegname: Rendiconti dell’Accademia Nazionale dei Lincei 40 (1985), pp. 225-233.

RoCCATI, A. - Sist, L.

2001 The archaeological expedition of the University of Rome La Sapienza in Egypt: M. CAsini (a cura di), One Hundred Years in Egypt: Paths of Italian Archaeology (Saggi di archeologia), Milano 2001, pp. 57-65. 
ROVERI, A

1967 La ceramica: Tamit (1964). Missione Archeologica in Egitto dell'Università di Roma (Istituto di Studi del Vicino Oriente. Serie Archeologica 14), Roma 1967, pp. 75-84.

SIST, L.

1979a A statue of Bakenkhons in the University Museum of Rome: W.F. ReINEKE (ed.), Acts, First International Congress of Egyptology, Berlin 1979, pp. 583-585.

1979b Stele Magica con Figurazione di Dea Serpente da Antinoe: Vicino Oriente II (1979), pp. 93-97.

1982 Alcune ceramiche decorate: Meroitic Studies, Meroitica 6 (1982), pp. 317-321.

1992a Un frammento di statua da Crocodilopoli: Vicino Oriente VIII (1992), pp. 49-68.

1992b The Reliefs of Tomb n. 27 at the Asasif: S. CuRTo S. - S. DONADONI - A.M. Donadoni ROVERI - B. Alberton (a cura di), Sesto Congresso Internazionale di Egittologia. Atti, Torino 1992, pp. 485-488.

1996 Materiale ceramico antinoita nel Museo del Vicino Oriente dell’Università di Roma La Sapienza: M.G. PICOZZI - F. CARINCI (a cura di), Studi in memoria di Lucia Guerrini. Vicino Oriente, Egeo, Grecia, Roma e mondo romano, tradizione dell'antico e collezionismo di antichità (Studi Miscellanei 30), Roma 1996, pp. 360-378.

2000 Natakamani e l'Ellenismo: alcune considerazioni sul palazzo B 1500 a Gebel Barkal: S. Russo (a cura di), Atti del V Convegno Nazionale di Egittologia e Papirologia (Firenze, 10-12 dic. 1999), Firenze 2000, pp. 253-257.

2005 Motivi ellenistici nell'architettura meroitica: nuove scoperte a Napata: Acta Nubica (Proceedings of the X International Conference of Nubia Studies, Rome 9-14 September 2002), Rome 2005, pp. 475-481.

2011 Preliminary notes on two royal buildings discovered in Napata: Vicino \& Medio Oriente XV (2011), pp. 159-166.

2012 La Chiesa nubiana di Sonqi Tino. Un caso di studio integrato sul patrimonio culturale: Scienze dell'Antichità 18 (2012), pp. 519-536.

2013 Osservazioni sul sarcofago del Museo del Vicino Oriente della Sapienza decorato con sfilata "demoniaca": I. BAGLIONI (a cura di), Monstra Costruzione e Percezione delle Entità Ibride e Mostruose nel Mediterraneo Antico, Roma 2013, pp. 73-83.

2014 La collezione egizia della Sapienza Università di Roma: il piatto di Hotepsekhemui: E.M. Ciampini - P. ZANovello (a cura di), Antichità egizie e Italia. Prospettive di ricerca e indagini sul campo (Antichistica 6. Serie di Studi Orientali 2), Venezia 2014, pp. 63-68.

SPALLANZANI ZIMMERMANN, A.

1974 Il cimitero protodinastico: Antinoe (1965-1968). Missione Archeologica in Egitto dell’Università di Roma (Istituto del Vicino Oriente. Serie Archeologica 21), Roma 1974, pp. 23-31.

TIRADRITTI, F.

1992 Stele di Amanitore e Arikankharor dal "Palazzo di Natakamani” al Gebel Barkal: Vicino Oriente VIII (1992), pp. 69-75. 


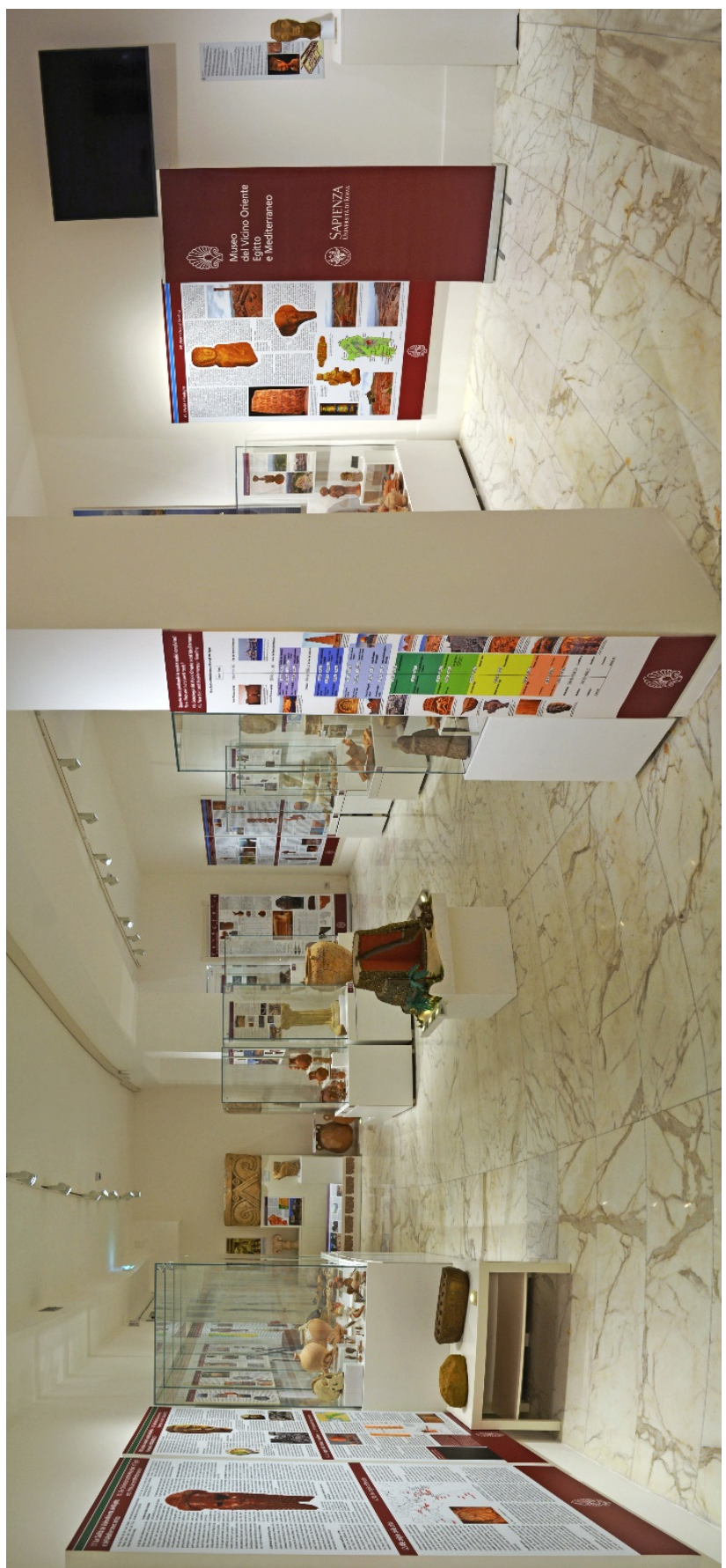

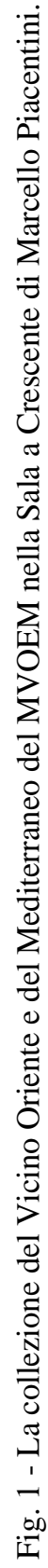




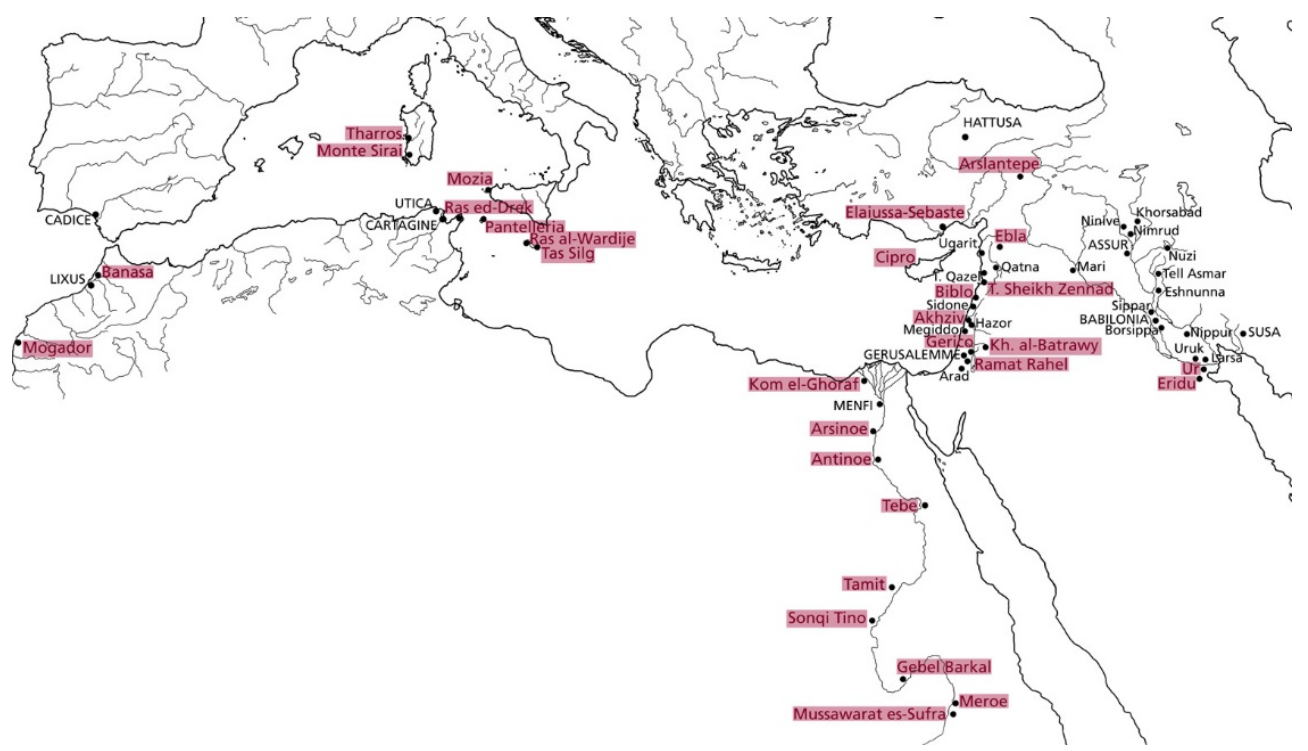

Fig. 2 - La mappa del Mediterraneo con evidenziati tutti i siti oggetto di ricerche i cui materiali sono conservati nel MVOEM.

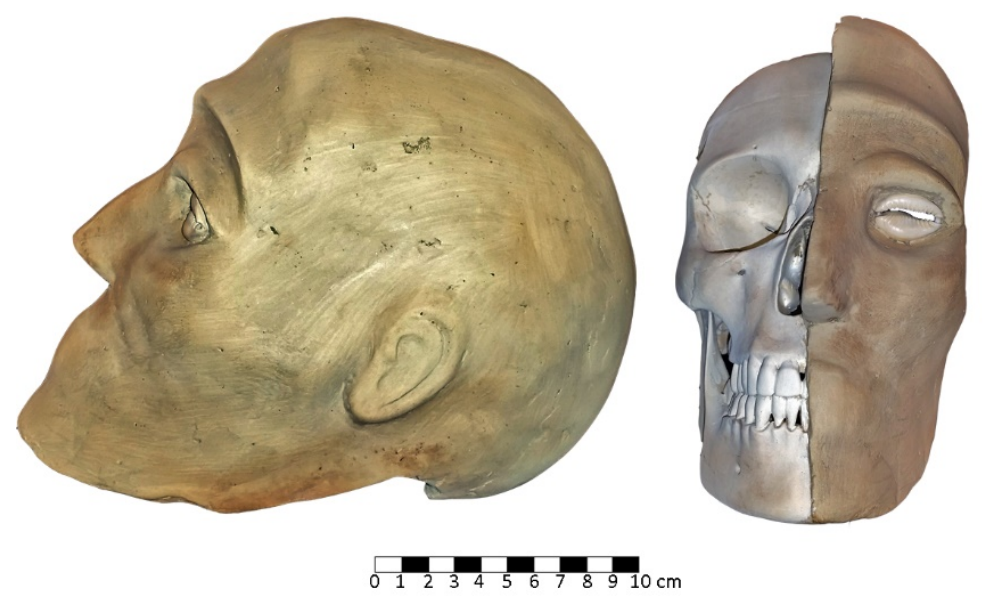

Fig. 3 - La riproduzione di teschio modellato del Neolitico Preceramico B di Gerico esposto nella vetrina 1 . 
Fig. 4 - Le copie di alcune tra le più significative cretule e di alcuni vasi ritrovati nel palazzo Tardo Uruk di Arslantepe.
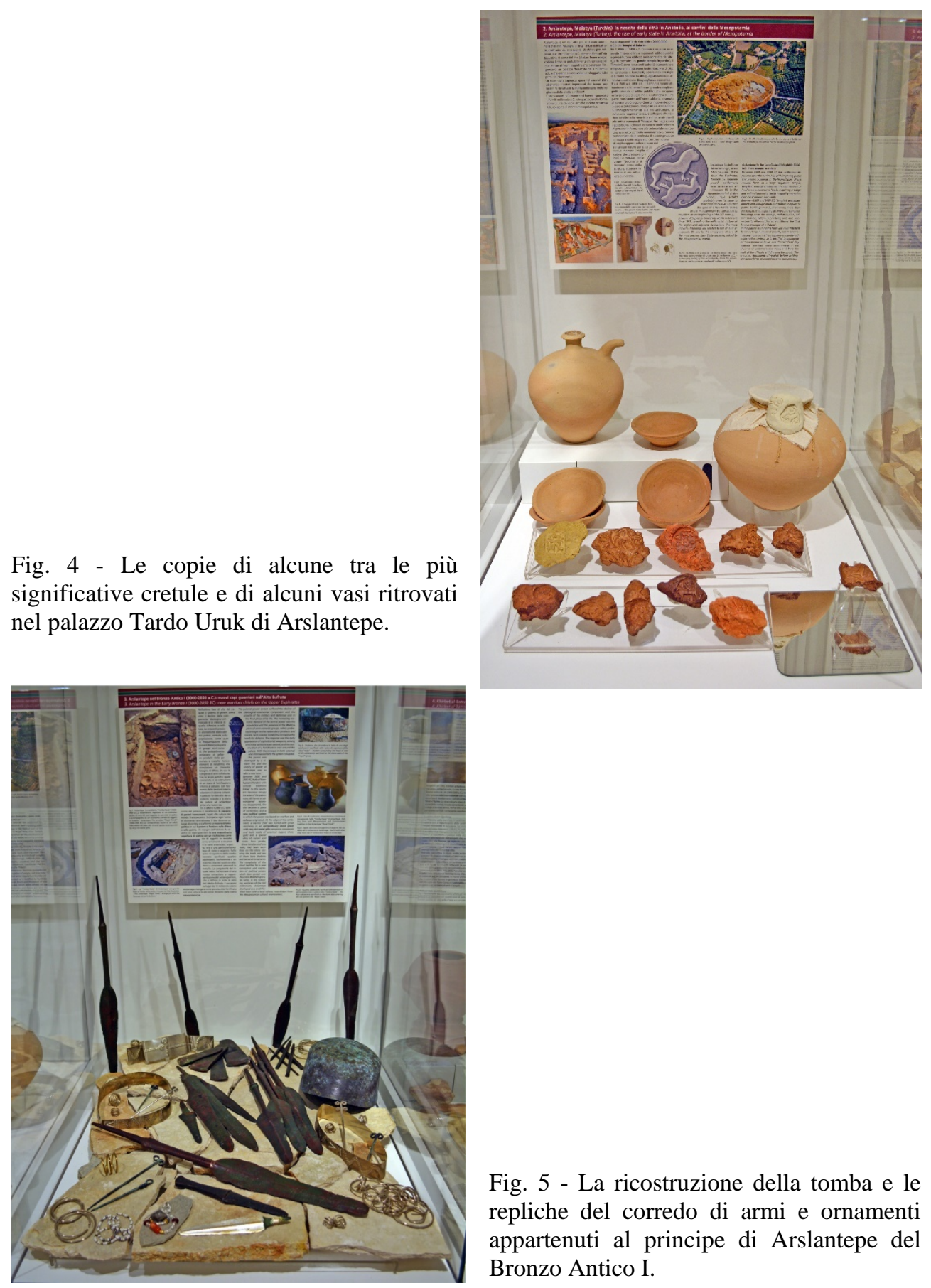

Fig. 5 - La ricostruzione della tomba e le repliche del corredo di armi e ornamenti appartenuti al principe di Arslantepe del Bronzo Antico I. 
Fig. 6 - Brocchette cipro-fenicie in ceramica Red Slip dipinte con motivi di cerchi concentrici neri e rossi.

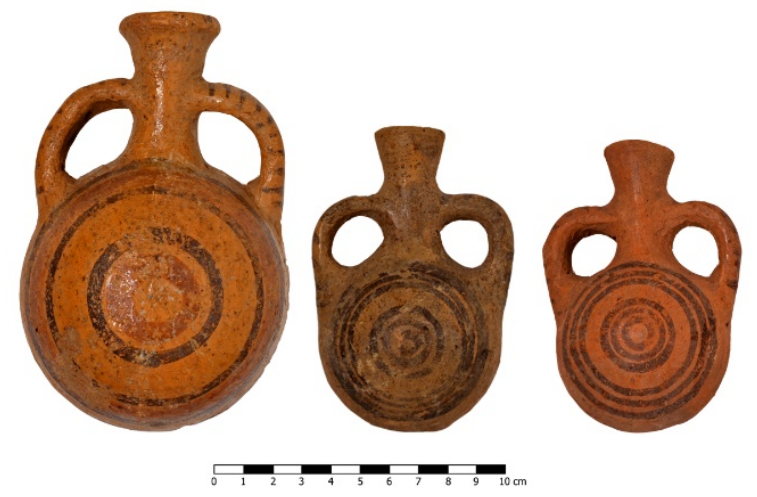

Fig. 7 - I reperti da Ramat Rahel dell'Età del Ferro esposti nella vetrina 7 .

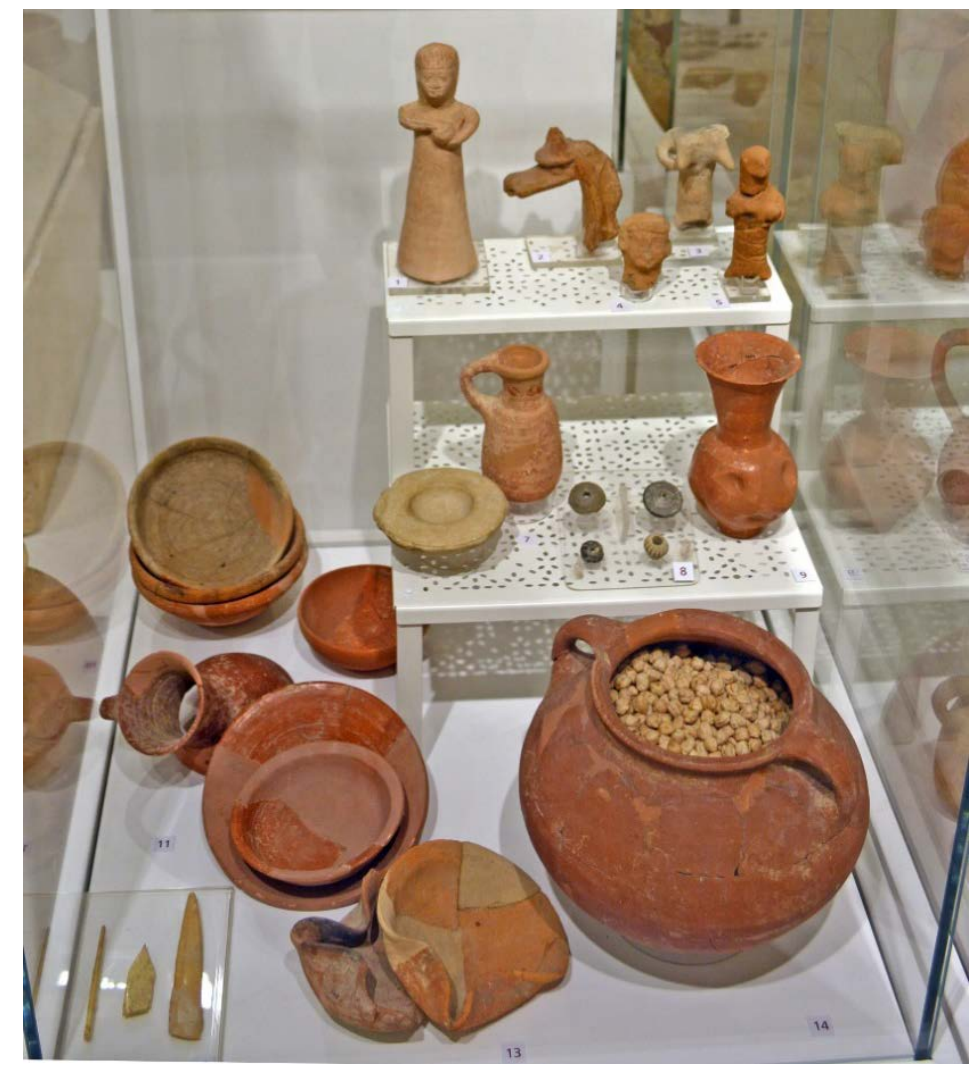




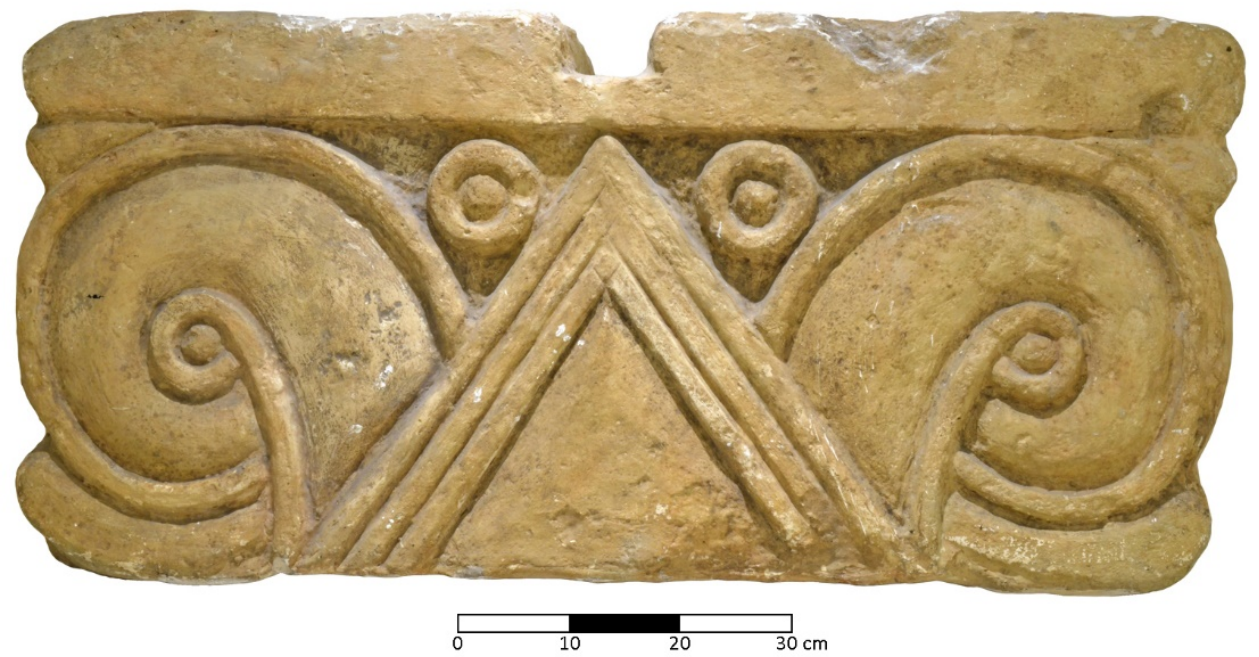

Fig. 8 - La ricostruzione completa di un capitello proto-eolico appartenuto alla facciata del palazzo interno alla Cittadella dell’VIII secolo a.C. di Ramat Rahel.

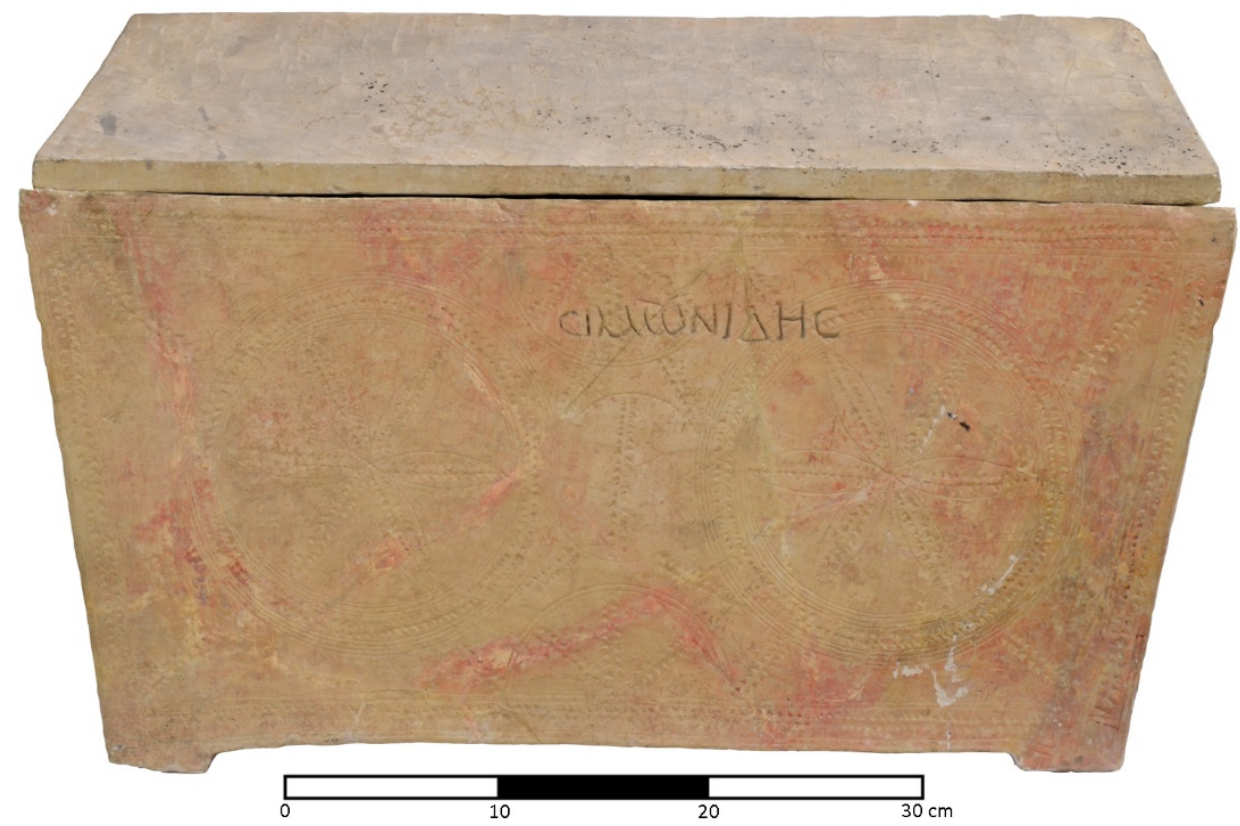

Fig. 9 - L'ossuario romano del I secolo d.C. con iscrizione $\Sigma \mathrm{IM} \Omega \mathrm{NI} \Delta \mathrm{H} \Sigma$ da Ramat Rahel. 


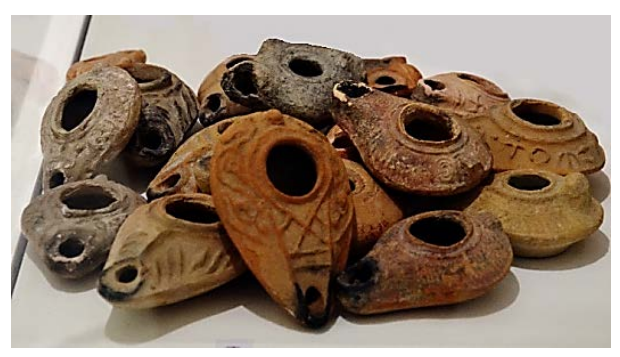

Fig. 10 - La collezione di lucerne di età bizantina e islamica da Ramat Rahel.

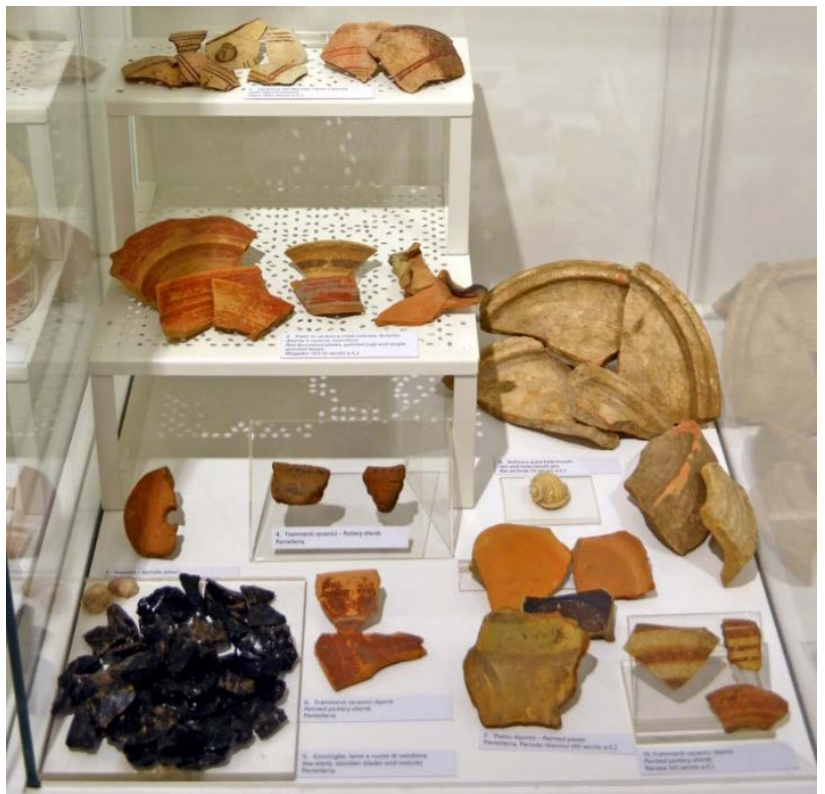

Fig. 11 - Alcuni reperti provenienti da Cipro, da Pantelleria, dalla Tunisia, dall'Algeria e dal Marocco esposti nella vetrina 11.

Fig. 12 - Testa di una statua schematica da Malta, III secolo a.C.

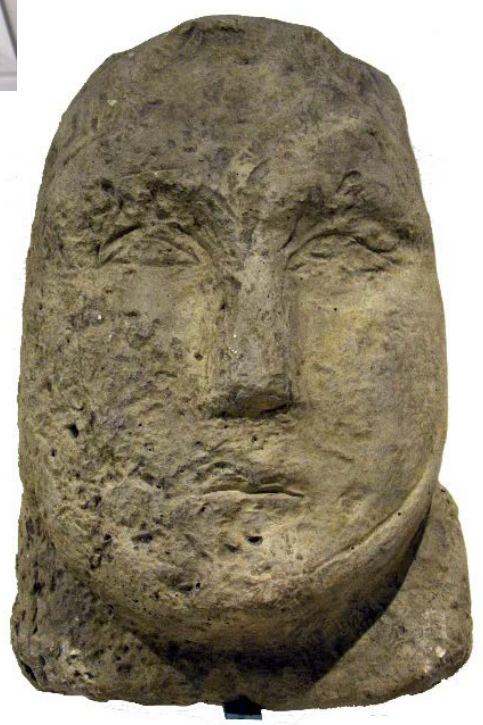




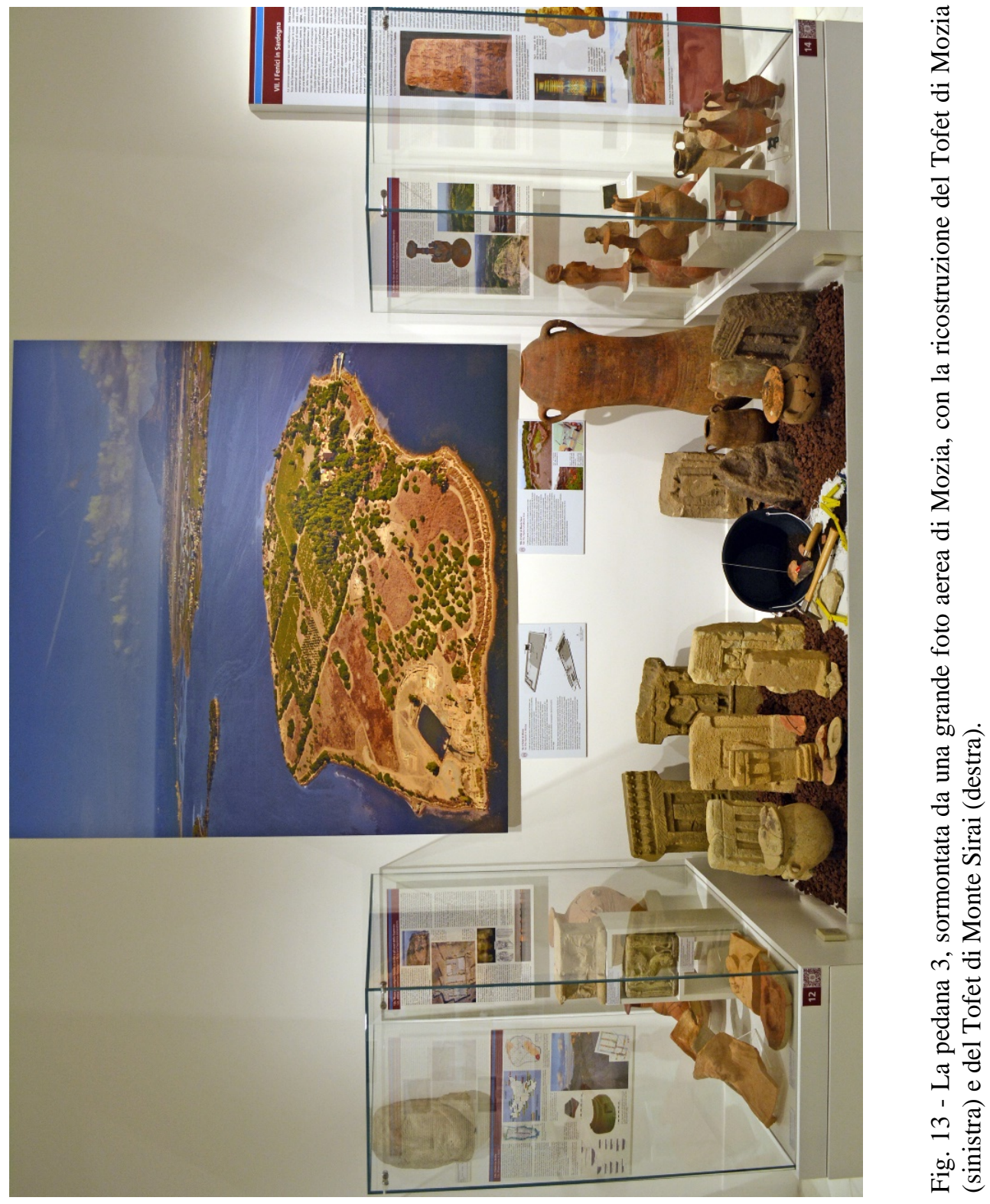


Fig. 14 - La palmetta eburnea rinvenuta nella necropoli di Monte Sirai, divenuta il simbolo del Museo.

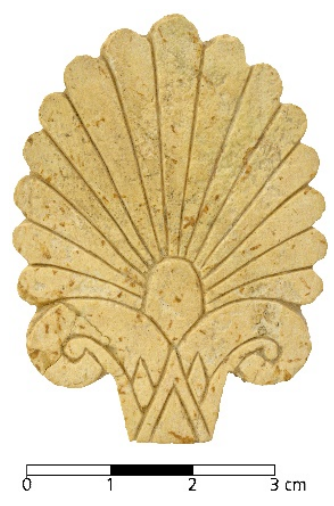

Fig. 15 - La copia in gesso del busto della statua della regina Tuya.

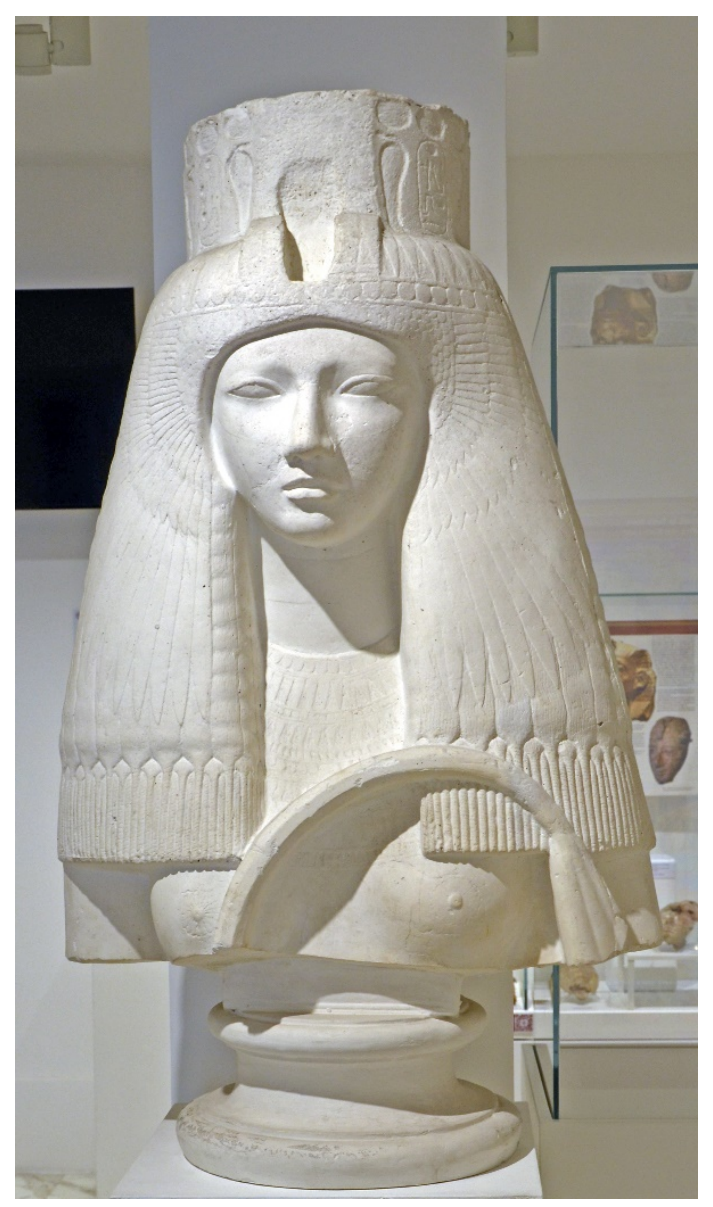




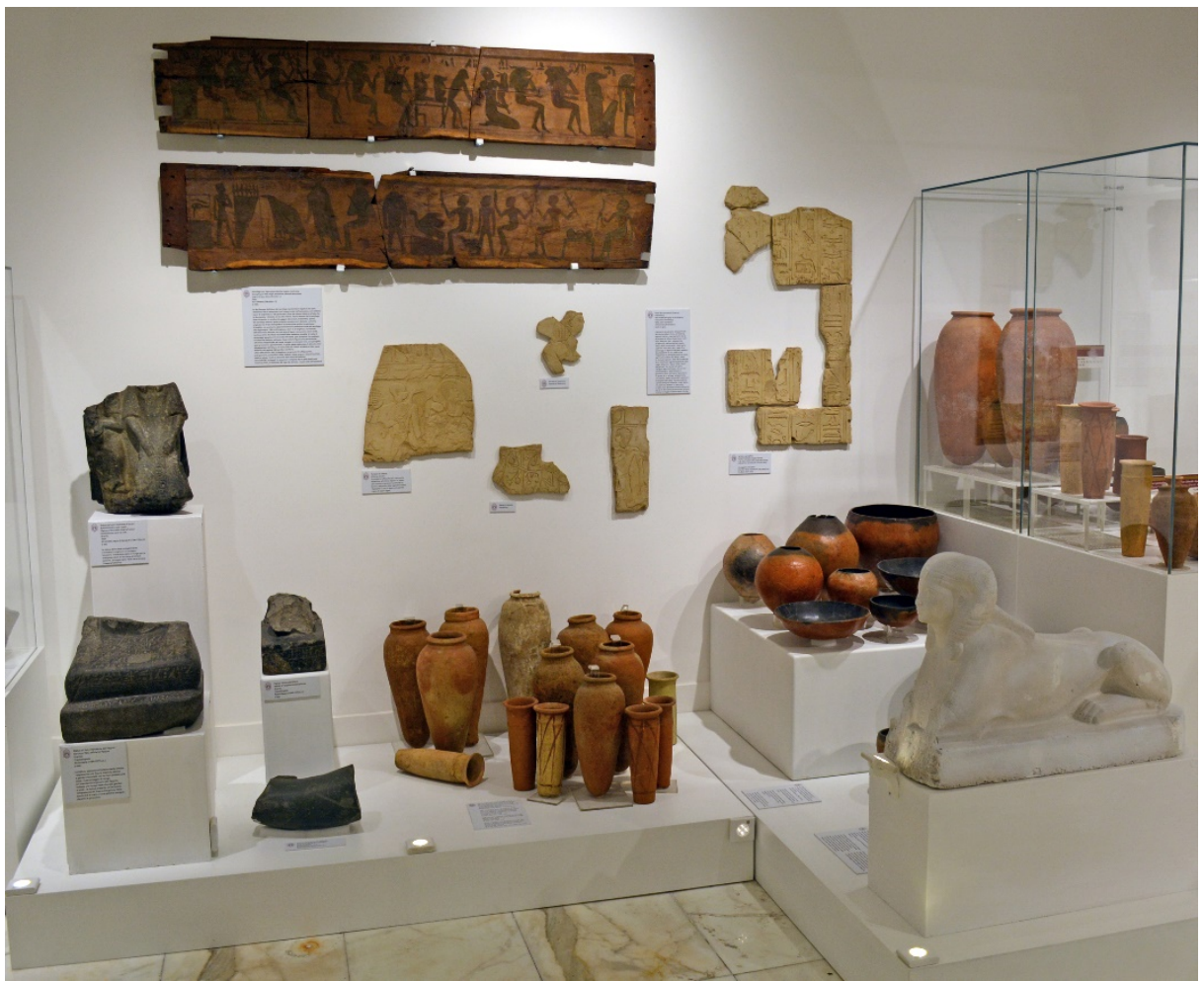

Fig. 16 - La pedana 4 sormontata da alcune copie di rilievi e iscrizioni geroglifiche della tomba di Sheshonq e dai due lati di un sarcofago ligneo dipinto, XXVI dinastia; alcune statue di funzionari di varia epoca (sinistra), i corredi delle tombe di Antinoe e Tamit (centro), e una copia in gesso della sfinge del regno di Thutmosis III (destra).

Fig. 17 - La riproduzione della testa di Sheshonq in un rilievo della Tomba 27 nella necropoli Assasif di Tebe, esposta nella vetrina 21.

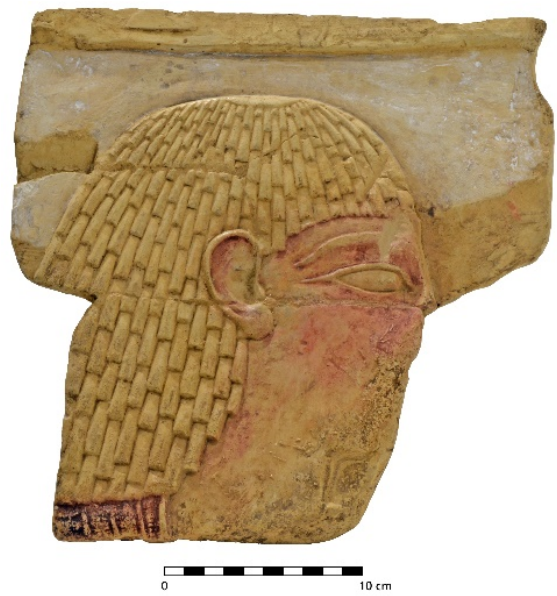




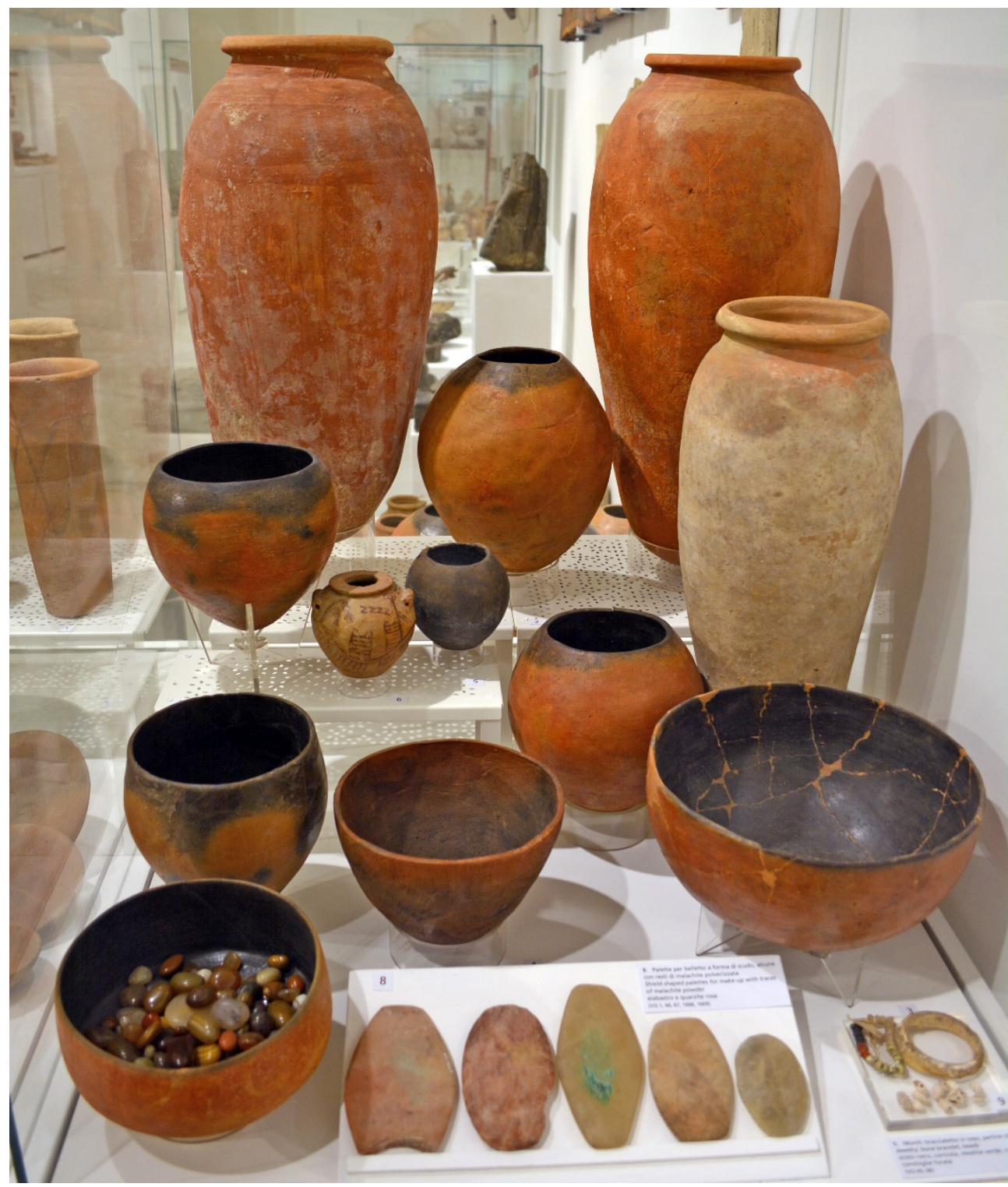

Fig. 18 - Alcuni elementi dei corredi della necropoli di Antinoe esposti nella vetrina 20. 

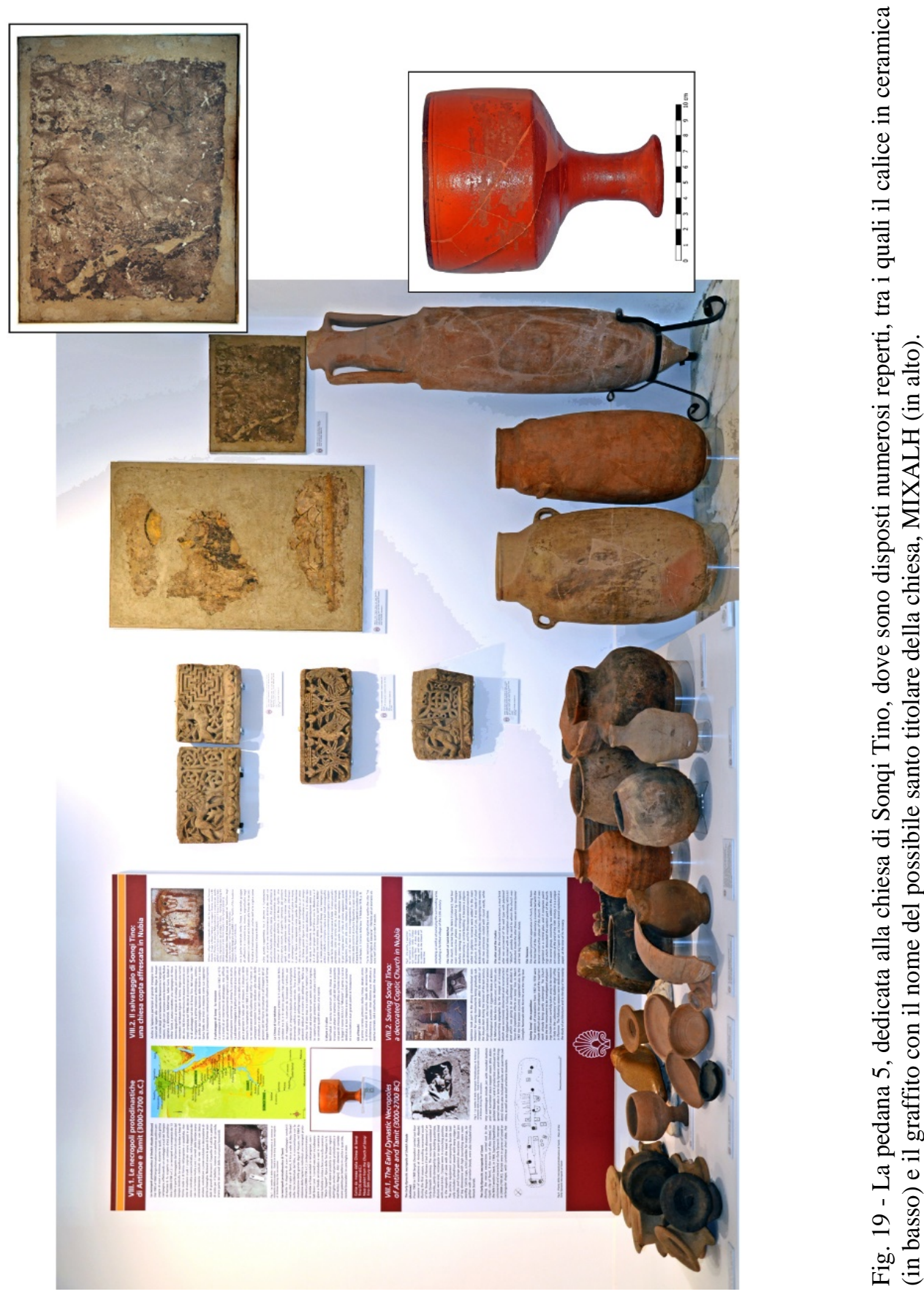


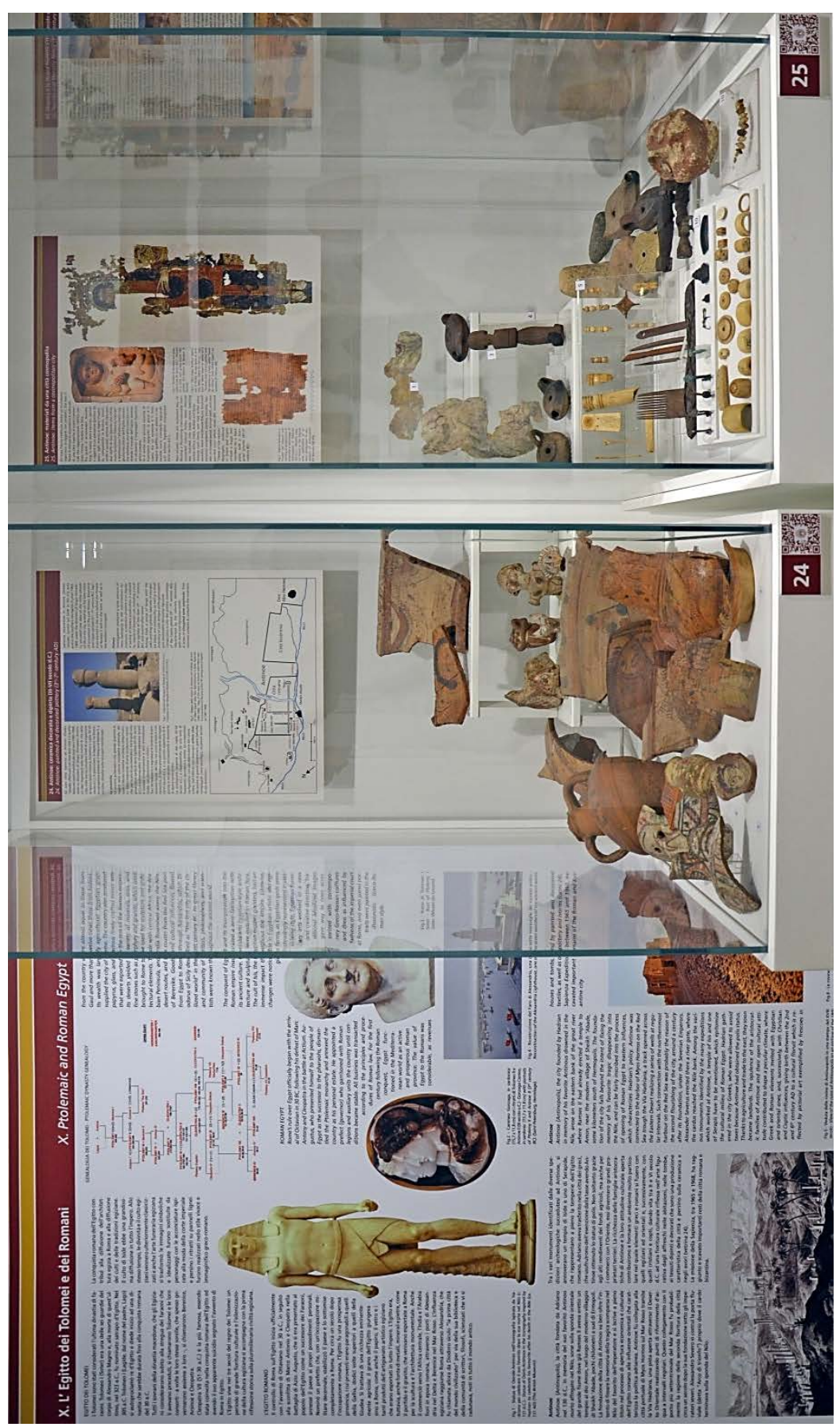

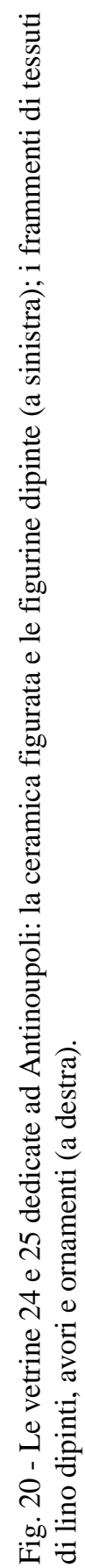




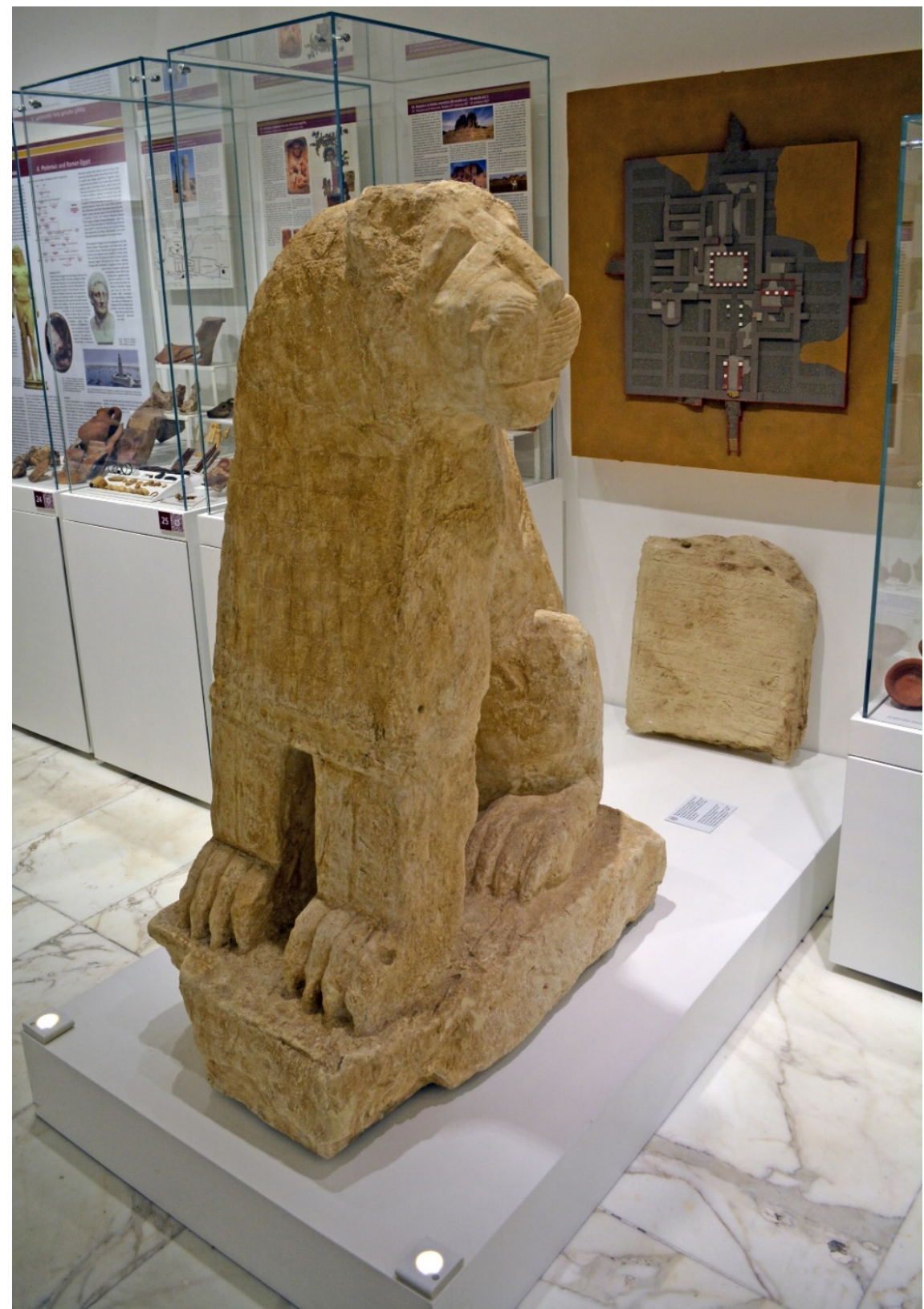

Fig. 21 - Il calco di un leone posto a guardia di uno degli ingressi del palazzo Jebel Barkal, in primo piano, e quello di una stele iscritta in meroitico, in secondo piano, sulla pedana 6; sulla parete il plastico del palazzo. 


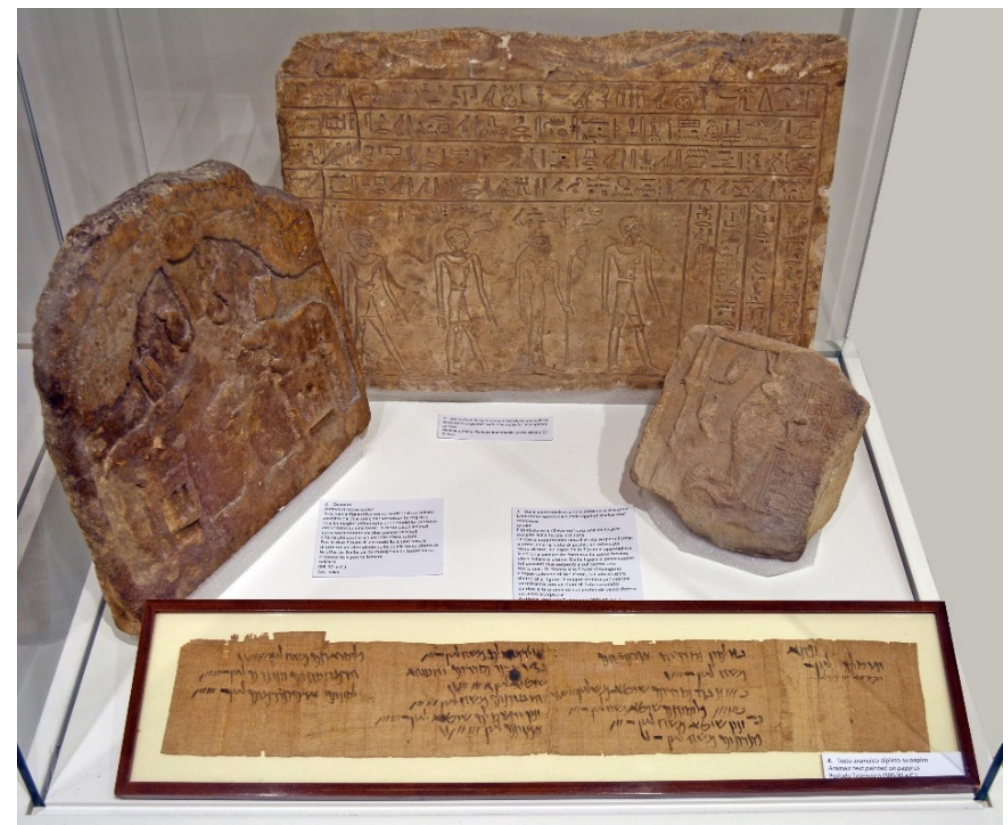

Fig. 22 - Le stele iscritte del Primo Periodo Intermedio (centro), d'età tolemaica (sinistra) e d'età romana (destra) e il papiro con un testo in aramaico del II secolo a.C., nella vetrina 28.

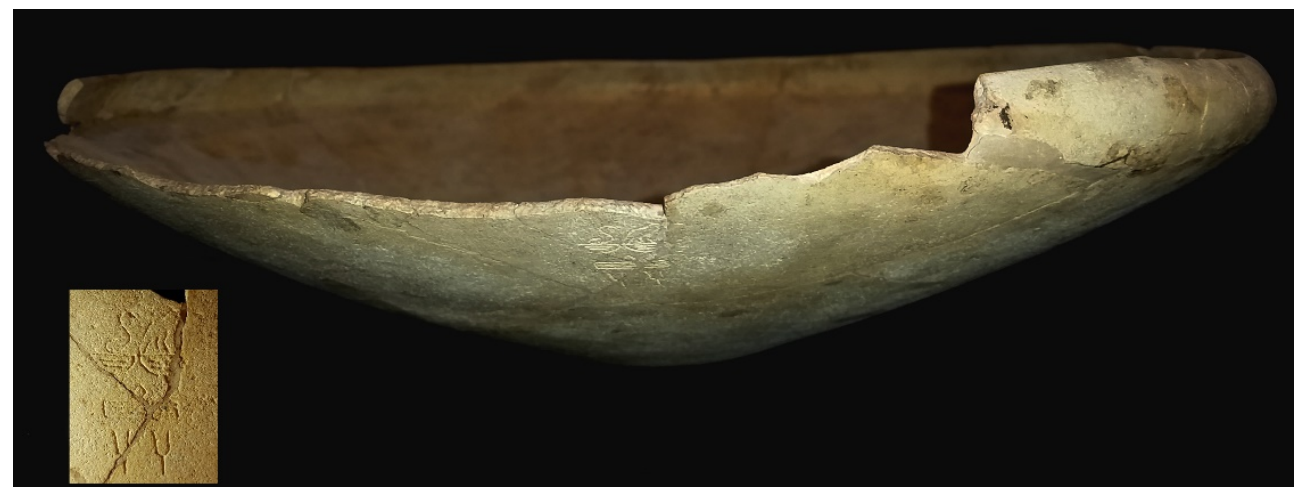

Fig. 23 - Il piatto in pietra tufacea giallo-ocra che reca, sotto l'orlo l'iscrizione del nome di Hotepsekhemwy. 


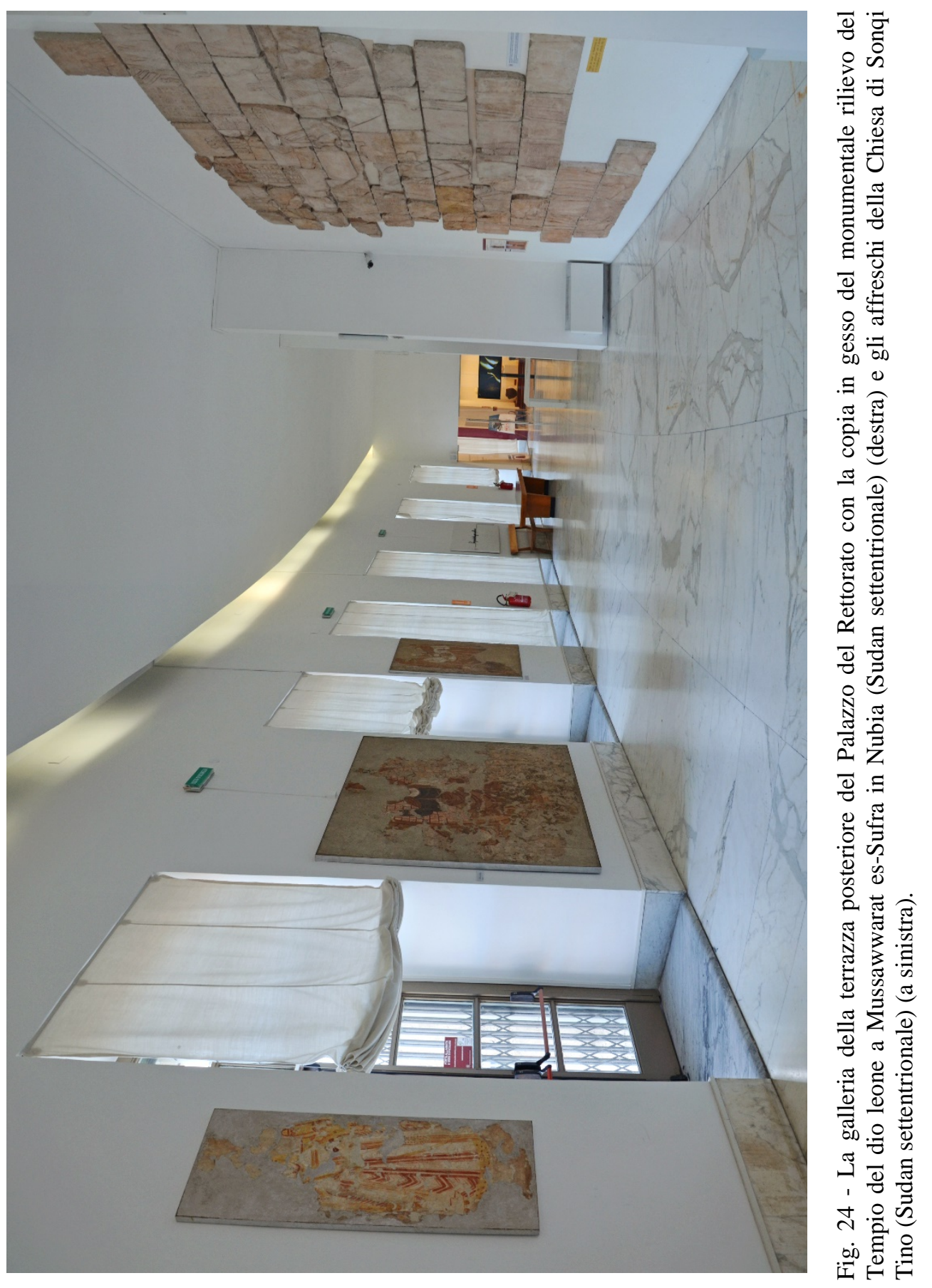




\section{SOMMARIO}

DipartimENTo SCIENZE DELl'ANTICHITÀ

SEZIONE di ORIENTALISTICA

\section{VICINO ORIENTE \\ XIX - 2015}

L. Nigro - Bethlehem in the Bronze and lron Ages
in the light of recent discoveries by the Palestinian MOTA-DACH

$\checkmark$. Pisaniello - Parallel passages among Hittite-Luwian rituals:

for the restoration of $K U B 35.146$

F. Spagnoli - Una testa di sileno in bronzo da Mozia

N. Chiaren

dall Area sacra del Kothon a Mozia

G. Labisi - al-Fudayn: an Umayyad residence in Northern Jordan

P. Buzi - Early Christianity in the Fayyūm: the new contribution of archaeology

I. Materia - Preliminary notes on the ware depicted on the ceiling

of the Cappella Palatina in Palermo

S. Autiero - Indian Ocean trade:

a reassessment of the pottery find
$\left(3^{\text {rd }}\right.$ century $B C-S^{S^{t h}}$ century $\left.A D\right)$

M.M. Jamhawi - N. Al-Shakarchi - I. Al-Hashimi

Assessment of tourists' satisfaction in the downtown of Amman

SCAVI E RICERCHE

L. Nigro - C. Fiaccavento - M. Jaradat - J. Yasine

A

L. Nigro - D. Montanari - M. Ghayyada - J. Yasine

A Middle Bronze and Iron Age necropolis near Bethlehem (Palestine)

L. Nigro - G. Ripepi - I. Hamdan - J. Yasine

15 Interim Report

and valorization of archaeological heritage

R. Francia - L'archivio di tavolette del complesso B-C-H di Büyükkale

organizazione degli archivi reali ittiti. Considerazioni preliminari

V. Pisaniello - La collezione di tavolette del complesso B-C-H di Büyükkale

T. De Vincenzi - L'archivio di tavolette del complesso B-C-H
sull'acropoli di Büyükkale

Museo del Vicino Oriente, Egitto e Mediterraneo

L. Nigro - Il nuovo allestimento del Museo del Vicino Oriente,

Egitto e Mediterraneo della Sapienza

D. Montanari - Bollettino delle attività del Museo del Vicino Oriente,

Egito e Meditraneo della Sapienza, anno 2015

RECENSIONI

A. Orsingher - E. PAPPA (2013), Early Iron Age Exchange in the West:

(Ancient Near Eastern Studies Supplement Series 43)

Leuven - Paris - Walpole 2013, MA.: Peeters $\frac{1}{2}$

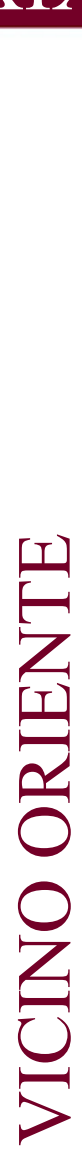

345

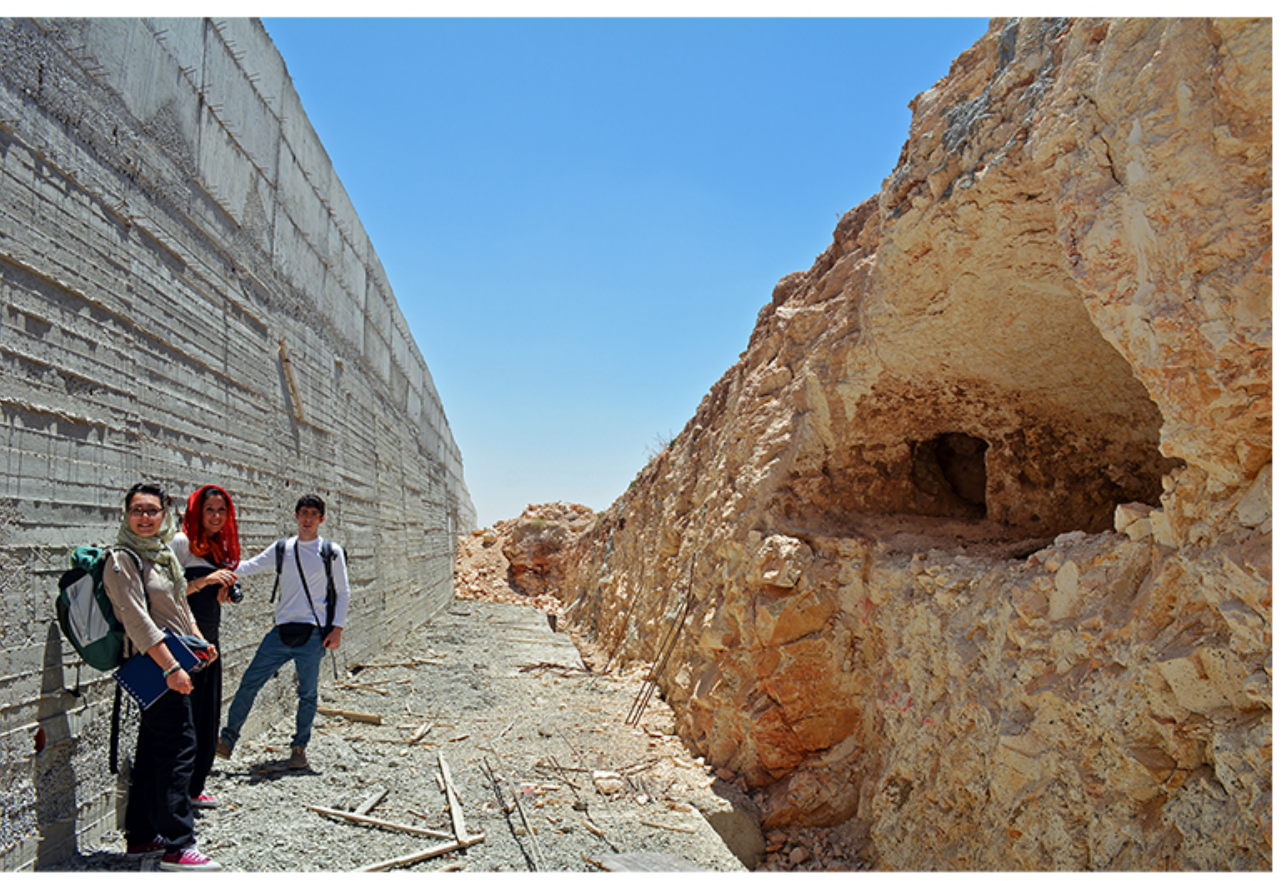

ROMA 2015 


\section{VICINO ORIENTE XIX - 2015}


VICINO ORIENTE

SAPIENZA UNIVERSITÀ DI ROMA

DIPARTIMENTO SCIENZE DELL'ANTICHITÀ

SEZIONE DI ORIENTALISTICA

Scientific Editor: Lorenzo Nigro

International Scientific Committee: Brian Rose, Frank Braemer, Mounir Fantar, Piero Bartoloni, Thomas Schaefer, Zeidan Kafafi

National Scientific Committee: Carlo Giovanni Cereti, Maria Vittoria Fontana, Sebastiano Tusa, Massimiliano Marazzi

Editorial Board: Daria Montanari, Chiara Fiaccavento

Tipografia: SK7 - Roma

ISSN 0393-0300

Rivista con comitato di referee

Journal with international referee system

www.lasapienzatojericho.it/SitoRivista/Journal/Rivista.php

In copertina: Tomba B9, necropoli di Khalet al-Jam’a (Betlemme). 
VICINO ORIENTE

SAPIENZA UNIVERSITÀ DI ROMA

DIPARTIMENTO SCIENZE DELL'ANTICHITÀ

SEZIONE DI ORIENTALISTICA

\section{SOMMARIO}

ARTICOLI

L. Nigro - Bethlehem in the Bronze and Iron Ages

in the light of recent discoveries by the Palestinian MOTA-DACH

V. Pisaniello - Parallel passages among Hittite-Luwian rituals:

for the restoration of KUB 35.146

F. Spagnoli - Una testa di sileno in bronzo da Mozia

N. Chiarenza - Una matrice per terrecotte con sileno dall'Area sacra del Kothon a Mozia

G. Labisi - al-Fudayn: an Umayyad residence in Northern Jordan

P. Buzi - Early Christianity in the Fayyūm: the new contribution of archaeology

I. Materia - Preliminary notes on the ware depicted on the ceiling

of the Cappella Palatina in Palermo

S. Autiero - Indian Ocean trade:

a reassessment of the pottery finds from a multidisciplinary point of view ( $3^{\text {rd }}$ century $B C-5^{\text {th }}$ century $\left.A D\right)$

M.M. Jamhawi - N. Al-Shakarchi - I. Al-Hashimi

Assessment of tourists' satisfaction in the downtown of Amman

SCAVI E RICERCHE

L. Nigro - C. Fiaccavento - M. Jaradat - J. Yasine Archaeology from A to Z: Abu Zarad, an ancient town in the heartland of Palestine

L. Nigro - D. Montanari - M. Ghayyada - J. Yasine

Khalet al-Jam'a. A Middle Bronze and Iron Age necropolis near Bethlehem (Palestine) 185 
VICINO ORIENTE

SAPIENZA UNIVERSITÀ DI ROMA

DIPARTIMENTO SCIENZE DELL'ANTICHITÀ

SEZIONE DI ORIENTALISTICA

L. Nigro - G. Ripepi - I. Hamdan - J. Yasine

The Jericho Oasis Archaeological Park - 2015 Interim Report.

Italian-Palestinian Cooperation for protection

and valorization of archaeological heritage

R. Francia - L'archivio di tavolette del complesso B-C-H di Büyükkale

e l'organizzazione degli archivi reali ittiti. Considerazioni preliminari

V. Pisaniello - La collezione di tavolette del complesso B-C-H di Büyükkale

T. De Vincenzi - L'archivio di tavolette del complesso B-C-H sull'acropoli di Büyükkale

Museo del Vicino ORIente, Egitto e MediterRaneo

L. Nigro - Il nuovo allestimento del Museo del Vicino Oriente,

Egitto e Mediterraneo della Sapienza

D. Montanari - Bollettino delle attività del Museo del Vicino Oriente,

Egitto e Mediterraneo della Sapienza, anno 2015

\section{RECENSIONI}

A. Orsingher - E. PAPPA (2013), Early Iron Age Exchange in the West:

Phoenicians in the Mediterranean and the Atlantic

(Ancient Near Eastern Studies Supplement Series 43),

Leuven - Paris - Walpole 2013, MA.: Peeters 